\title{
HIGGS BUNDLES AND HIGHER TEICHMÜLLER SPACES
}

\author{
OSCAR GARCÍA-PRADA
}

\begin{abstract}
This paper is a survey on the role of Higgs bundle theory in the study of higher Teichmüller spaces. Recall that the Teichmüller space of a compact surface can be identified with a certain connected component of the moduli space of representations of the fundamental group of the surface into PSL $(2, \mathbb{R})$. Higher Teichmüller spaces correspond to special components of the moduli space of representations when one replaces $\operatorname{PSL}(2, \mathbb{R})$ by a real non-compact semisimple Lie group of higher rank. Examples of these spaces are provided by the Hitchin components for split real groups, and maximal Toledo invariant components for groups of Hermitian type. More recently, the existence of such components has been proved for $\mathrm{SO}(p, q)$, in agreement with the conjecture of Guichard and Wienhard relating the existence of higher Teichmüller spaces to a certain notion of positivity on a Lie group that they have introduced. We review these three different situations, and end up explaining briefly the conjectural general picture from the point of view of Higgs bundle theory.
\end{abstract}

\section{INTRODUCTION}

Given a compact surface $S$ of genus $g \geq 2$, consider the moduli space of representations of the fundamental group of $S$ in $\operatorname{SL}(2, \mathbb{R})$. Attached to such a representation there is an integer invariant $d$, which by Milnor [Mil58] satisfies the bound $|d| \leq g-1$. In the maximal case $d=g-1$ ( or $d=1-g$ ) the moduli space has $2^{2 g}$ connected components, all of which are homeomorphic to $\mathbb{R}^{6 g-6}$ and can be identified to the Teichmüller space of $S$. All the representations in these components are Fuchsian, that is, discrete and faithful. When passing to the adjoint group $\operatorname{PSL}(2, \mathbb{R})$ all these components get identified.

Higher Teichmüller spaces appear when one replaces $\operatorname{SL}(2, \mathbb{R})$ by certain real Lie groups of higher rank. These spaces have been studied from several points of view. In this paper we will focus on the role played by the theory of Higgs bundles. This powerful theory, introduced by Hitchin [Hit87], is very useful in identifying these spaces and studying their topology, in particular counting the number of connected components. To do this, one chooses a complex structure on $S$, making it into a compact Riemann surface $X$. Given a semisimple real Lie group $G$, with a choice of a maximal compact subgroup $H \subset G$, one has a Cartan decomposition of the Lie algebra of $G$, given by $\mathfrak{g}=\mathfrak{h} \oplus \mathfrak{m}$, where $\mathfrak{h}$ is the Lie algebra of $H$ and $\mathfrak{m}$ is the orthogonal complement of $\mathfrak{h}$ with respect to the Killing form of $\mathfrak{g}$. A $G$-Higgs bundle over $X$ is a pair $(E, \varphi)$ consisting of a holomorphic $H^{\mathbb{C}}$-bundle $E$ over $X$ and a holomorphic section $\varphi$ of the bundle $E\left(\mathfrak{m}^{\mathbb{C}}\right) \otimes K$,

Date: 6 January 2019.

2000 Mathematics Subject Classification. Primary 14H60; Secondary 57R57, 58D29.

The author is partially supported by the Spanish MINECO under the ICMAT Severo Ochoa grant No. SEV-2011-0087, and under grant No. MTM2013-43963-P. . 
where $E\left(\mathfrak{m}^{\mathbb{C}}\right)$ is the vector bundle associated to $E$ via the isotropy representation $H^{\mathbb{C}} \rightarrow$ $\mathrm{GL}\left(\mathfrak{m}^{\mathbb{C}}\right)$, and $K$ is the canonical line bundle of $X$ - the holomorphic cotangent bundle of $X$. The non-abelian Hodge correspondence proved by Hitchin [Hit87], Donaldson [Don87], Simpson [Sim88, Sim92], Corlette [Cor88] and others (see [GGM09]) establishes a homeomorphism between the moduli space $\mathcal{M}(G)$ of polystable $G$-Higgs bundles over $X$ and the moduli space $\mathcal{R}(G)$ of reductive representations of the fundamental group of $S$ in $G$.

In [Hit92] Hitchin constructed components of the moduli space of representations $\mathcal{R}(G)$ when $G$ is the split real form of a complex simple Lie group $G^{\mathbb{C}}$ which, like Teichmüller space, are homeomorphic to a real vector space, in this case of dimension $\operatorname{dim} G(2 g-2)$. Originally referred by him as generalized Teichmüller components, they are now called Hitchin components. Such components appear as sections of the Hitchin fibration for the moduli space of $G^{\mathbb{C}}$-Higgs bundles. This fibration is defined by the map $h: \mathcal{M}\left(G^{\mathbb{C}}\right) \rightarrow B\left(G^{\mathbb{C}}\right)$ sending a $G^{\mathbb{C}}$-Higgs bundle $(E, \varphi)$ to $\left(p_{1}(\varphi), \ldots, p_{r}(\varphi)\right)$, where $p_{1}, \ldots, p_{r}$ is a basis of the ring of $G^{\mathbb{C}}$-invariant homogeneous polynomials of $\mathfrak{g}^{\mathbb{C}}$. Hence $B\left(G^{\mathbb{C}}\right)=\oplus_{i=1}^{r} H^{0}\left(X, K^{d_{i}}\right)$, where $d_{i}$ is the degree of $p_{i}$. These degrees are indepedent of the basis and depend only on the group. The Hitchin components can then be identified with the vector space $B\left(G^{\mathbb{C}}\right)$ which has half the dimension of $\mathcal{M}\left(G^{\mathbb{C}}\right)$. An alternative (equivalent) way of definining the Hitchin components is by directly considering the Hitchin map for the Lie group $G$. In fact this exists for any semisimple Lie group $G$. The Hitchin map in this case $h: \mathcal{M}(G) \rightarrow B(G)$ is obtained by applying a basis of the ring of $H^{\mathbb{C}}$-invariant polynomials of $\mathfrak{m}^{\mathbb{C}}$. One can construct in this general situation a section of $h$, but only when $G$ is split does this define a component of $\mathcal{M}(G)$ - when $G$ is split $B(G)=B\left(G^{\mathbb{C}}\right)$ (see [GPR18]).

The Hitchin components consist entirely of representations of $\pi_{1}(S)$ in $G$ that can be deformed to a representation that factors through a Fuchsian representation $\pi_{1}(S) \rightarrow$ $\mathrm{SL}(2, \mathbb{R})$, where $\mathrm{SL}(2, \mathbb{R}) \rightarrow G$ is the irreducible representation of $\mathrm{SL}(2, \mathbb{R})$ in $G$, which always exists when $G$ is a split real form. One may need to consider $\operatorname{PSL}(2, \mathbb{R})$ if the group $G$ is of adjoint type. In this case, like for $\operatorname{PSL}(2, \mathbb{R})$, the Hitchin component is unique. It follows from the work of Labourie [Lab06] and Fock and Goncharov [FG06] that, similarly to the Teichmüller component for $\operatorname{PSL}(2, \mathbb{R})$, all the representations in a Hitchin component are discrete and faithful. In his paper [Hit92] Hitchin already posed the question on the relation of his generalized Teichmüller components with geometric structures. A geometric interpretation of these components for $G=\mathrm{SL}(3, \mathbb{R})$ has been given by Choi-Goldman [ChG93] and more recently for more general split groups by Labourie [Lab06] Fock-Goncharov [FG06], and Guichard-Wienhard [GW08].

The group $\operatorname{SL}(2, \mathbb{R})$ is of course a split real form of $\operatorname{SL}(2, \mathbb{C})$ but it is also a group of Hermitian type. For such a group $G$, the symmetric space $G / H$ is Kähler. The symmetric space $\mathrm{SL}(2, \mathbb{R}) / \mathrm{SO}(2)$ can be identified with the Poincaré upper-half plane. Another direction in which Teichmüller space can be generalized is by considering a higher rank non-compact simple Lie group $G$ of Hermitian type with finite centre. The Lie algebra of a group of this type is one of the following: $\mathfrak{s u}(p, q), \mathfrak{s} \mathfrak{o}^{*}(2 n), \mathfrak{s p}(2 n, \mathbb{R})$, $\mathfrak{s o}(2, n), \mathfrak{e}_{6}^{-14}$ and $\mathfrak{e}_{7}^{-25}$ (we are using Helgason's notation [Hel01]). In this situation the centre $\mathfrak{z}$ of $\mathfrak{h}$ is isomorphic to $\mathbb{R}$, and the adjoint action of a special element $J \in \mathfrak{z}$ defines an almost complex structure on $\mathfrak{m}=T_{o}(G / H)$, where $o \in G / H$ corresponds to 
the coset $H$, making the symmetric space $G / H$ into a Kähler manifold. The almost complex structure ad $(J)$ gives a decomposition $\mathfrak{m}^{\mathbb{C}}=\mathfrak{m}^{+}+\mathfrak{m}^{-}$in $\pm i$-eigenspaces, which is $H^{\mathbb{C}}$-invariant. An immediate consequence of this decomposition for a $G$-Higgs bundle $(E, \varphi)$ is that it gives a bundle decomposition $E\left(\mathfrak{m}^{\mathbb{C}}\right)=E\left(\mathfrak{m}^{+}\right) \oplus E\left(\mathfrak{m}^{-}\right)$and hence the Higgs field decomposes as $\varphi=\left(\varphi^{+}, \varphi^{-}\right)$, where $\varphi^{+} \in H^{0}\left(X, E\left(\mathfrak{m}^{+}\right) \otimes K\right)$ and $\varphi^{-} \in H^{0}\left(X, E\left(\mathfrak{m}^{-}\right) \otimes K\right)$.

Another important ingredient of $G$-Higgs bundles for this class of groups is the Toledo invariant. This is a topological invariant attached to a $G$-Higgs bundle $(E, \varphi)$, in fact attached to $E$. It is defined by considering a special character $\chi_{T}$ of $\mathfrak{h}^{\mathbb{C}}$ called the Toledo character. If this lifts to a character $\tilde{\chi}_{T}$ of $H^{\mathbb{C}}$, we consider the associated line bundle $E\left(\tilde{\chi}_{T}\right)$, and define the Toledo invariant $\tau$ of $(E, \varphi)$ as

$$
\tau=\tau(E):=\operatorname{deg}\left(E\left(\tilde{\chi}_{T}\right)\right) .
$$

Otherwise one can show that there is a rational number $q_{T}$ such $q_{T} \chi_{T}$ lifts to a character $\tilde{\chi}_{T}$, and one can define $\tau=\tau(E):=\frac{1}{q_{T}} \operatorname{deg}\left(E\left(\tilde{\chi}_{T}\right)\right)$. A crucial fact is given by the Milnor-Wood inequality: The moduli space $\mathcal{M}(G)$ is empty unless

$$
|\tau| \leq \operatorname{rk}(G / H)(2 g-2) .
$$

This was proved on a case by case basis for the classical groups [Hit87, Got01, BGG03, BGG06, BGG15, GGM13] and in general in [BGR17]. The Toledo invariant of $(E, \varphi)$ coincides with the classical Toledo invariant of a representation of the fundamental group in $G$, which is defined by integrating over $X$ the pull-back of the Kähler form of the Bergman metric of $G / H$, via a $\rho$-equivariant map $f: \widetilde{X} \rightarrow G / H$ determined by $\rho$. The map $f$ can be taken to be harmonic if the representation is reductive, and hence corresponding to a polystable $G$-Higgs bundle. In the context of representations the inequality $|\tau| \leq \operatorname{rk}(G / H)(2 g-2)$, goes back to Milnor [Mil58], who studies the case $G=\mathrm{SL}(2, \mathbb{R})$, as mentioned above, and was proved in various cases in [Woo71, Dup78, DT87, CO03], and in general by Burger-Iozzi-Wienhard [BIW10].

The higher Teichmüller spaces appear when the Toledo invariant is maximal, i.e., when $|\tau|=\operatorname{rk}(G / H)(2 g-2)$, and the symmetric space $G / H$ is of tube type. A geometric characterization of the tube type condition is given in terms of the Shilov boundary of the Harish-Chandra bounded symmetric domain realization of $G / H$. We say that $G / H$ is of tube type if the Shilov boundary of the corresponding bounded symmetric domain is a symmetric space $H / H^{\prime}$ (of compact type). If this is the case, this domain is biholomorphic to a 'tube' over the symmetric cone $\Omega:=H^{*} / H^{\prime}$ — the non-compact symmetric space dual of the Shilov boundary. The simplest example of this is given by $G=\mathrm{SL}(2, \mathbb{R}) \cong \mathrm{SU}(1,1)$, where the bounded symmetric domain is the Poincaré disc and the tube realization is the Poincare upper half plane (these are of course related by the classical Cayley transform). Sometimes we will say that the group $G$ is of tube type if the symmetric space $G / H$ is of tube type. The groups of tube type have Lie algebra given by $\mathfrak{s u}(p, q)$ with $p=q, \mathfrak{s o}^{*}(2 n)$ with $n$ even, $\mathfrak{s p}(2 n, \mathbb{R}), \mathfrak{s o}(2, n)$ and $\mathfrak{e}_{7}^{-25}$. While those of non-tube type are $\mathfrak{s u}(p, q)$ with $p \neq q, \mathfrak{s o}^{*}(2 n)$ with $n$ odd, and $\mathfrak{e}_{6}^{-14}$.

The subvariety $\mathcal{R}_{\max }(G) \subset \mathcal{R}(G)$ of maximal representations (and corresponding subvariety $\left.\mathcal{M}_{\max }(G) \subset \mathcal{M}(G)\right)$ in the tube case has special significance since, as proved in [BIW10], it consists entirely of discrete and faithful representations, like the Hitchin 
components in the split case. As in this case, all the representations in $\mathcal{R}_{\max }(G)$ are Anosov in the sense of Labourie [Lab06, BILW05], and are related to geometric structures of various kinds [GW12].

From the Higgs bundle point of view the tube-type condition plays a fundamental role in constructing a bijective correspondence - called Cayley correspondence - between the moduli space of maximal Higgs bundles $\mathcal{M}_{\max }(G)$ and the moduli space of $K^{2}$ twisted $H^{*}$-Higgs bundles over $X$, where $H^{*} \subset H^{\mathbb{C}}$ is the non-compact dual of $H$, described above. These are defined like Higgs bundles except that the twisting is by $K^{2}$ instead of $K$. An immediate consequence of this correspondence is the existence of new topological invariants for maximal Higgs bundles which are hidden a priori, and which play a crucial role in determining the number of connected components of $\mathcal{M}_{\max }(G)$.

Up to a finite cover, there is only one group which is both split and of Hermitian type, and in fact of tube type. This is the symplectic group $\operatorname{Sp}(2 n, \mathbb{R})$. The tube realization is the Siegel upper half space. The Hitchin representations are actually maximal but, except for the case $n=1$, there are maximal components that are not Hitchin components (see [Got01, GGM13]).

Maximal Higgs bundles, and hence maximal representations, in the non-tube case present also very interesting phenomena. It turns out that in this case the dimension of the moduli space of maximal $G$-Higgs bundles is smaller than expected, since up to a compact factor every maximal representation reduces to a representation in a maximal subgroup of tube type. This rigidity phenomenon is very rare in the context of surface groups (see [Tol89, Her91, BGG03, BGG06, BIW10, BGG15, BGR17]), and it is conjectured to happen only for Hermitian groups of non-tube type [KP14].

For a while, split real groups and Hermitian groups were the only cases providing examples of higher Teichmüller spaces. However, gradually there was evidence that there may be other groups. Groups under scrutiny were the special orthogonal groups of signature $(p, q)$. Of course, for certain values of $p$ and $q$ the group is split or Hermitian, but for most values is neither. One such evidence came from the work of Guichard and Wienhard [GW16]. They defined a notion of positivity for certain Lie groups and for surface group representations into those groups. The notion of positivity for a representation, which refines Labourie's Anosov condition, is open and they conjecture that is also closed, hence detecting connected components of the moduli space of representations. They showed that apart from the split real forms and the real forms of Hermitian type, the only other non-exceptional groups which allow positive representations are the groups locally isomorphic to $\mathrm{SO}(p, q)$ for $1<p<q$. Indeed, Collier [Col17] has proved existence of special components containing positive representations for $\mathrm{SO}(n, n+1)$. Although this group is split, the Collier components are different to the Hitchin ones. More recently the existence of special components for $\operatorname{SO}(p, q)$ with $1<p<q$ containing positive representations has been shown in [ABC $+18, \mathrm{ABC}+18 \mathrm{~b}]$.

After discribing the basic ingredients in the theory of Higgs bundles and the three different situations where higher Teichmüller spaces emerge (split real forms, real forms of Hermitian type and $\mathrm{SO}(p, q)$ ), we finish the paper with some comments on the conjectural general picture. This seems to relate positive representations, and hence higher Teichmüller spaces, to components of the moduli space for which there exists a Cayley 
correspondence that generalizes the one for Hermitian groups of tube type and the one for the special components in the moduli space of $\mathrm{SO}(p, q)$-Higgs bundles. Indeed there is also a Cayley correspondence for the Hitchin components. This seems to be the way in which positivity is manifesting from the point of view of Higgs bundle theory. A full study of this is under investigation in [BCGGO], including the exceptional groups for which there is a notion of positivity. As shown in [GW16] these are real forms of $F_{4}$, $E_{6}, E_{7}$ and $E_{8}$ whose restricted root system is of type $F_{4}$.

As mentioned above, we have focused here on the point of view provided by the theory of Higgs bundles, and offering just a very partial picture. For other approaches, and additional references, one may see the very nice survey papers [BIW14, Wien18]. We have not touched at all, the case of non-compact surfaces for which there is also a very rich higher Teichmüller theory. This situation is studied for example in [FG06, BIW10]. The Higgs bundle approach for the case of surfaces with punctures is very little developped. This involves the theory of parabolic Higgs bundles. In [BGM15] a nonabelian Hodge correspondence is established between parabolic G-Higgs bundles and representations of the fundamental group of the punctured surface with fixed conjugacy classes around the puntures, where $G$ is any real form of a complex reductive Lie group. This generalizes the work of Simpson [Sim90] when $G=\operatorname{GL}(n, \mathbb{C})$. Although the split and the Hermitian cases are briefly described in [BGM15], much work has to be done along the lines of the compact case to study the higher Teichmüller spaces.

Acknowledgements. The author would like to thank Athanase Papadopoulos for his kind invitation to contribute to this volume with this paper, and his patience in the delivery. He also wishes to thank his collaborators in the works on which this paper is based, and Nigel Hitchin for inspiration, suggestions and many discussions over the years.

\section{Higgs BundLES}

2.1. Basic definitions. Following Knapp [Kna96, VII.2], we define a real reductive group is a 4 -tuple $(G, H, \theta, B)$ where

(1) $G$ is a real Lie group with reductive Lie algebra $\mathfrak{g}$.

(2) $H<G$ is a maximal compact subgroup.

(3) $\theta$ is a Lie algebra involution of $\mathfrak{g}$ inducing an eigenspace decomposition

$$
\mathfrak{g}=\mathfrak{h} \oplus \mathfrak{m}
$$

where $\mathfrak{h}=\operatorname{Lie}(H)$ is the $(+1)$-eigenspace for the action of $\theta$, and $\mathfrak{m}$ is the $(-1)$ eigenspace.

(4) $B$ is a $\theta$ - and $\operatorname{Ad}(G)$-invariant non-degenerate bilinear form, with respect to which $\mathfrak{h} \perp_{B} \mathfrak{m}$ and $B$ is negative definite on $\mathfrak{h}$ and positive definite on $\mathfrak{m}$.

(5) The multiplication map $H \times \exp (\mathfrak{m}) \rightarrow G$ is a diffeomorphism.

(6) $G$ acts by inner automorphisms on the complexification $\mathfrak{g}^{\mathbb{C}}$ of its Lie algebra via the adjoint representation.

In this section we fix a real reductive Lie group $(G, H, \theta, B)$. We have $[\mathfrak{m}, \mathfrak{m}] \subset \mathfrak{h}$, $[\mathfrak{m}, \mathfrak{h}] \subset \mathfrak{h}$. Complexifying the isotropy representation $H \rightarrow \operatorname{Aut}(\mathfrak{m})$, we obtain the representation $\operatorname{Ad}: H^{\mathbb{C}} \rightarrow \operatorname{Aut}\left(\mathfrak{m}^{\mathbb{C}}\right)$. When $G$ is semisimple we take $B$ to be the Killing 
form. In this case $B$ and a choice of a maximal compact subgroup $H$ determine a Cartan decomposition.

Let $X$ be a compact Riemann surface of genus $g$.

A $G$-Higgs bundle on $X$ consists of a holomorphic principal $H^{\mathbb{C}}$-bundle $E$ together with a holomorphic section $\varphi \in H^{0}\left(X, E\left(\mathfrak{m}^{\mathbb{C}}\right) \otimes K\right)$, where $E\left(\mathfrak{m}^{\mathbb{C}}\right)$ is the associated vector bundle with fibre $\mathfrak{m}^{\mathbb{C}}$ via the complexified isotropy representation, and $K$ is the canonical line bundle of $X$.

If $G$ is compact, $H=G$ and $\mathfrak{m}=0$. A $G$-Higgs bundle is hence simply a holomorphic principal $G^{\mathbb{C}}$-bundle. If $G=H^{\mathbb{C}}$, where $H$ is a maximal compact subgroup of $G, \mathfrak{m}=i \mathfrak{h}$, and a $G$-Higgs bundle is a principal $G$-bundle together with a section $\varphi \in H^{0}(X, E(\mathfrak{g}) \otimes$ $K)$, where $E(\mathfrak{g})$ is the adjoint bundle. This is the original definition for complex Lie groups given by Hitchin in [Hit87b].

Let $G^{\prime}$ be a reductive subgroup of $G$. A maximal compact subgroup of $G^{\prime}$ is given by $H^{\prime}=H \cap G^{\prime}$ and we can take a compatible Cartan decomposition, in the sense that $\mathfrak{h}^{\prime} \subset \mathfrak{h}$ and $\mathfrak{m}^{\prime} \subset \mathfrak{m}$. Moreover, the isotropy representation of $H^{\prime}$ is the restriction of the isotropy representation Ad of $H$. We say that the structure group of a $G$-Higgs bundle $(E, \varphi)$ reduces to $G^{\prime}$ when there is a reduction of the structure group of the underlying $H^{\mathbb{C}}$-bundle to $H^{\prime \mathbb{C}}$, given by a subbundle $E_{\sigma}$, and the Higgs field $\varphi \in H^{0}\left(X, E\left(\mathfrak{m}^{\mathbb{C}}\right) \otimes K\right)$ belongs to $H^{0}\left(X, E_{\sigma}\left(\mathfrak{m}^{\prime \mathbb{C}}\right) \otimes K\right)$.

2.2. Stability of $G$-Higgs bundles. There is a notion of stability for $G$-Higgs bundles (for details see [GGM09]). To explain this we consider the parabolic subgroups of $H^{\mathbb{C}}$ defined for $s \in i \mathfrak{h}$ as

$$
P_{s}=\left\{g \in H^{\mathbb{C}}: e^{t s} g e^{-t s} \text { is bounded as } t \rightarrow \infty\right\} .
$$

When $H^{\mathbb{C}}$ is connected every parabolic subgroup is conjugate to one of the form $P_{s}$ for some $s \in i \mathfrak{h}$, but this is not the case necessarily when $H^{\mathbb{C}}$ is non-connected. A Levi subgroup of $P_{s}$ is given by $L_{s}=\left\{g \in H^{\mathbb{C}}: \operatorname{Ad}(g)(s)=s\right\}$, and any Levi subgroup is given by $p L_{s} p^{-1}$ for $p \in P_{s}$. Their Lie algebras are given by

$$
\begin{aligned}
\mathfrak{p}_{s} & =\left\{Y \in \mathfrak{h}^{\mathbb{C}}: \operatorname{Ad}\left(e^{t s}\right) Y \text { is bounded as } t \rightarrow \infty\right\}, \\
\mathfrak{l}_{s} & =\left\{Y \in \mathfrak{h}^{\mathbb{C}}: \operatorname{ad}(Y)(s)=[Y, s]=0\right\} .
\end{aligned}
$$

We consider the subspaces

$$
\begin{aligned}
& \mathfrak{m}_{s}=\left\{Y \in \mathfrak{m}^{\mathbb{C}}: \operatorname{Ad}\left(e^{t s}\right) Y \text { is bounded as } t \rightarrow \infty\right\} \\
& \mathfrak{m}_{s}^{0}=\left\{Y \in \mathfrak{m}^{\mathbb{C}}: \operatorname{Ad}\left(e^{t s}\right) Y=Y \text { for every } t\right\} .
\end{aligned}
$$

One has that $\mathfrak{m}_{s}$ is invariant under the action of $P_{s}$ and $\mathfrak{m}_{s}^{0}$ is invariant under the action of $L_{s}$.

Remark 2.1. The subalgebra $\mathfrak{m}_{s}$ is the non-compact part of the parabolic subalgebra of $\mathfrak{g}^{\mathbb{C}}$ defined by $s \in i \mathfrak{h}$. Define $\widetilde{\mathfrak{p}}_{s}=\left\{Y \in \mathfrak{g}^{\mathbb{C}} \mid \operatorname{Ad}\left(e^{t s}\right) Y\right.$ is bounded as $\left.t \rightarrow \infty\right\}$. We have that $\mathfrak{p}_{s}=\widetilde{\mathfrak{p}}_{s} \cap \mathfrak{h}^{\mathbb{C}}$ and $\mathfrak{m}_{s}=\widetilde{\mathfrak{p}}_{s} \cap \mathfrak{m}^{\mathbb{C}}$. Analogously, define

$$
\widetilde{\mathfrak{l}}_{s}=\left\{Y \in \mathfrak{g}^{\mathbb{C}} \mid \operatorname{Ad}\left(e^{t s}\right) Y=Y \text { for every } t\right\} .
$$

Then $\mathfrak{l}_{s}=\widetilde{\mathfrak{l}}_{s} \cap \mathfrak{h}^{\mathbb{C}}$ and $\mathfrak{m}_{s}^{0}=\widetilde{\mathfrak{l}}_{s} \cap \mathfrak{m}^{\mathbb{C}}$. 
An element $s \in i \mathfrak{h}$ defines a character $\chi_{s}$ of $\mathfrak{p}_{s}$ since $\left\langle s,\left[\mathfrak{p}_{s}, \mathfrak{p}_{s}\right]\right\rangle=0$. Conversely, by the isomorphism $\left(\mathfrak{p}_{s} /\left[\mathfrak{p}_{s}, \mathfrak{p}_{s}\right]\right)^{*} \cong \mathfrak{z}_{s}^{*}$, where $\mathfrak{z}_{s}$ is the centre of the Levi subalgebra $\mathfrak{l}_{s}$, a character $\chi$ of $\mathfrak{p}_{s}$ is given by an element in $\mathfrak{z}_{s}^{*}$, which gives, via the invariant metric, an element $s_{\chi} \in \mathfrak{z}_{s} \subset i \mathfrak{h}$. When $\mathfrak{p}_{s} \subset \mathfrak{p}_{s_{\chi}}$, we say that $\chi$ is an antidominant character of $\mathfrak{p}$. When $\mathfrak{p}_{s}=\mathfrak{p}_{s_{\chi}}$ we say that $\chi$ is a strictly antidominant character. Note that for $s \in i \mathfrak{h}$, $\chi_{s}$ is a strictly antidominant character of $\mathfrak{p}_{s}$.

Let now $(E, \varphi)$ be a $G$-Higgs bundle over $X$, and let $s \in i \mathfrak{h}$. Let $P_{s}$ be defined as above. For $\sigma \in \Gamma\left(E\left(H^{\mathbb{C}} / P_{s}\right)\right)$ a reduction of the structure group of $E$ from $H^{\mathbb{C}}$ to $P_{s}$, we define the degree relative to $\sigma$ and $s$, or equivalently to $\sigma$ and $\chi_{s}$, as follows. When a real multiple $\mu \chi_{s}$ of the character exponentiates to a character $\tilde{\chi}_{s}$ of $P_{s}$, we compute the degree as

$$
\operatorname{deg}(E)(\sigma, s)=\frac{1}{\mu} \operatorname{deg}\left(E_{\sigma}\left(\tilde{\chi}_{s}\right)\right) .
$$

This condition is not always satisfied, but one shows ([GGM09, Sec. 4.6]) that the antidominant character can be expressed as a linear combination of characters of the centre and fundamental weights, and there exists an integer multiple $m$ of the characters of the centre and the fundamental weights exponentiating to the group, so we can define the degree.

An alternative definition of the degree can be given in terms of the curvature of connections using Chern-Weil theory. This definition is more natural when considering gauge-theoretic equations as we do below. For this, define $H_{s}=H \cap L_{s}$ and $\mathfrak{h}_{s}=\mathfrak{h} \cap \mathfrak{l}_{s}$. Then $H_{s}$ is a maximal compact subgroup of $L_{s}$, so the inclusions $H_{s} \subset L_{s}$ is a homotopy equivalence. Since the inclusion $L_{s} \subset P_{s}$ is also a homotopy equivalence, given a reduction $\sigma$ of the structure group of $E$ to $P_{s}$ one can further restrict the structure group of $E$ to $H_{s}$ in a unique way up to homotopy. Denote by $E_{\sigma}^{\prime}$ the resulting $H_{s}$ principal bundle. Consider now a connection $A$ on $E_{\sigma}^{\prime}$ and let $F_{A} \in \Omega^{2}\left(X, E_{\sigma}^{\prime}\left(\mathfrak{h}_{s}\right)\right.$ be its curvature. Then $\chi_{s}\left(F_{A}\right)$ is a 2 -form on $X$ with values in $i \mathbb{R}$, and

$$
\operatorname{deg}(E)(\sigma, s):=\frac{i}{2 \pi} \int_{X} \chi_{s}\left(F_{A}\right) .
$$

We define the subalgebra $\mathfrak{h}_{\text {ad }}$ as follows. Consider the decomposition $\mathfrak{h}=\mathfrak{z}+[\mathfrak{h}, \mathfrak{h}]$, where $\mathfrak{z}$ is the centre of $\mathfrak{h}$, and the isotropy representation ad $=\operatorname{ad}: \mathfrak{h} \rightarrow \operatorname{End}(\mathfrak{m})$. Let $\mathfrak{z}^{\prime}=\operatorname{ker}\left(\operatorname{ad}_{\mid \mathfrak{z}}\right)$ and take $\mathfrak{z}^{\prime \prime}$ such that $\mathfrak{z}=\mathfrak{z}^{\prime}+\mathfrak{z}^{\prime \prime}$. Define the subalgebra $\mathfrak{h}_{\text {ad }}:=\mathfrak{z}^{\prime \prime}+[\mathfrak{h}, \mathfrak{h}]$. The subindex ad denotes that we have taken away the part of the centre $\mathfrak{z}$ acting trivially via the isotropy representation ad.

Remark 2.2. For groups of Hermitian type, $\mathfrak{z}^{\prime}=0$ since an element both in $\mathfrak{z}$ and ker(ad) belongs to the centre of $\mathfrak{g}$, which is zero, as $\mathfrak{g}$ is semisimple. Hence $\mathfrak{h}_{\text {ad }}=\mathfrak{h}$.

With $L_{s} \mathfrak{m}_{s}$ and $\mathfrak{m}_{s}^{0}$ defined as above. We have the following.

Definition 2.3. Let $\alpha \in i \mathfrak{z} \subset \mathfrak{z}^{\mathbb{C}}$. We say that a $G$-Higgs bundle $(E, \varphi)$ is:

$\alpha$-semistable if for any $s \in i \mathfrak{h}$ and any holomorphic reduction $\sigma \in \Gamma\left(E\left(H^{\mathbb{C}} / P_{s}\right)\right)$ such that $\varphi \in H^{0}\left(X, E_{\sigma}\left(\mathfrak{m}_{s}\right) \otimes K\right)$, we have that $\operatorname{deg}(E)(\sigma, s)-\chi_{s}(\alpha) \geq 0$.

$\alpha$-stable if for any $s \in i \mathfrak{h}_{\mathrm{ad}}$ and any holomorphic reduction $\sigma \in \Gamma\left(E\left(H^{\mathbb{C}} / P_{s}\right)\right)$ such that $\varphi \in H^{0}\left(X, E_{\sigma}\left(\mathfrak{m}_{s}\right) \otimes K\right)$, we have that $\operatorname{deg}(E)(\sigma, s)-\chi_{s}(\alpha)>0$. 
$\alpha$-polystable if it is $\alpha$-semistable and for any $s \in i \mathfrak{h}_{\mathrm{ad}}$ and any holomorphic reduction $\sigma \in \Gamma\left(E\left(H^{\mathbb{C}} / P_{s}\right)\right)$ such that $\varphi \in H^{0}\left(X, E_{\sigma}\left(\mathfrak{m}_{s}\right) \otimes K\right)$ and $\operatorname{deg}(E)(\sigma, s)-\chi_{s}(\alpha)=0$, there is a holomorphic reduction of the structure group $\sigma_{L} \in \Gamma\left(E_{\sigma}\left(P_{s} / L_{s}\right)\right)$ to a Levi subgroup $L_{s}$ such that $\varphi \in H^{0}\left(X, E_{\sigma_{L}}\left(\mathfrak{m}_{s}^{0}\right) \otimes K\right) \subset H^{0}\left(X, E_{\sigma}\left(\mathfrak{m}_{s}\right) \otimes K\right)$.

Remark 2.4. We may define a real group $G_{L_{s}}=\left(L_{s} \cap H\right) \exp \left(\mathfrak{m}_{s}^{0} \cap \mathfrak{m}\right)$ with maximal compact subgroup the compact real form $L_{s} \cap H$ of the complex group $L_{s}$ and $\mathfrak{m}_{s}^{0} \cap \mathfrak{m}$ as isotropy representation. Thus, an $\alpha$-polystable $G$-Higgs bundle reduces to an $\alpha$ polystable $G_{L_{s}}$-Higgs bundle since $\varphi$ belongs $H^{0}\left(X, E_{\sigma_{L}}\left(\mathfrak{m}_{s}^{0}\right) \otimes K\right)$.

Remark 2.5. We can replace $K$ in the definition of $G$-Higgs bundle by any holomorphic line bundle $L$ on $X$. More precisely, an $L$-twisted $G$-Higgs bundle $(E, \varphi)$ consists of a principal $H^{\mathbb{C}}$-bundle $E$, and a holomorphic section $\varphi \in H^{0}\left(X, E\left(\mathfrak{m}^{\mathbb{C}}\right) \otimes L\right)$. We reserve the name $G$-Higgs bundle for the $K$-twisted case. The stability criteria are as in Definition 3.5, replacing $K$ by $L$.

Remark 2.6. For $G$ semisimple, the notion of $\alpha$-stability with $\alpha \neq 0$ only makes sense for groups of Hermitian type, since $\alpha$ belongs to the centre of $\mathfrak{h}$, which is not zero if and only if the centre of a maximal compact subgroup $H$ is non-discrete, i.e., if $G$ is of Hermitian type. For reductive groups which are not of Hermitian type, $\alpha$-stability makes sense, but there is only one value of $\alpha$ for which the condition is not void. This value is fixed by the topology of the principal bundle (see [GO14] for details).

Remark 2.7. When $H^{\mathbb{C}}$ is connected, as mentioned above, every parabolic subgroup of $H^{\mathbb{C}}$ is conjugate to one of the form $P_{s}$ for $s \in i \mathfrak{h}$. In this situation, we can formulate the stability conditions in Definition 2.3 in terms of any parabolic subgroup $P \subset H^{\mathbb{C}}$, replacing $s$ by $s_{\chi}$, for any antidominant character $\chi$ of $\mathfrak{h}^{\mathbb{C}}$.

Two $G$-Higgs bundles $(E, \varphi)$ and $\left(E^{\prime}, \varphi^{\prime}\right)$ are isomorphic if there is an isomorphism $f: E \rightarrow E^{\prime}$ such that $\varphi^{\prime}=f^{*} \varphi$, where $f^{*}$ is the map $E\left(\mathfrak{m}^{\mathbb{C}}\right) \otimes K \rightarrow E^{\prime}\left(\mathfrak{m}^{\mathbb{C}}\right) \otimes K$ induced by $f$.

The moduli space of $\alpha$-polystable $G$-Higgs bundles $\mathcal{M}^{\alpha}(G)$ is defined as the set of isomorphism classes of $\alpha$-polystable $G$-Higgs bundles on $X$. When $\alpha=0$ we simply denote $\mathcal{M}(G):=\mathcal{M}^{0}(G)$.

Remark 2.8. Similarly, we can define the moduli space of $\alpha$-polystable $L$-twisted $G$-Higgs bundles which will be denoted by $\mathcal{M}_{L}^{\alpha}(G)$.

These moduli spaces have the structure of a complex analytic varieties, as one can see by the standard slice method, which gives local models via the so-called Kuranishi map (see, e.g., [Kob87]). When $G$ is algebraic and under fairly general conditions, the moduli spaces $\mathcal{M}^{\alpha}(G)$ can be constructed by geometric invariant theory and hence are complex algebraic varieties. The work of Schmitt [Sch08] deals with the construction of the moduli space of $L$-twisted $G$-Higgs bundles for $G$ a real reductive Lie group. This construction generalizes the constructions of the moduli space of $G$-Higgs bundles done by Ramanathan [Ram96] when $G$ is compact ( $G$-Higgs bundles in this case are simply $G^{\mathbb{C}}$-principal bundles), and by Simpson [Sim94, Sim95] when $G$ is a complex reductive algebraic (see also $[\mathrm{Nit} 91]$ for $G=\mathrm{GL}(n, \mathbb{C})$ ). 
Remark 2.9. If $G=\operatorname{GL}(n, \mathbb{C})$, we recover the original notions of Higgs bundle and stability introduced by Hitchin [Hit87]. A $G=\mathrm{GL}(n, \mathbb{C})$-Higgs bundle is given by a holomorphic vector bundle $V:=E\left(\mathbb{C}^{n}\right)$ - associated to a principal $\mathrm{GL}(n, \mathbb{C})$-bundle $E$ via the standard representation — and a homomorphism

$$
\Phi: V \longrightarrow V \otimes K \text {. }
$$

The Higgs bundle $(V, \Phi)$ is stable if

$$
\frac{\operatorname{deg} V^{\prime}}{\operatorname{rank} V^{\prime}}<\frac{\operatorname{deg} V}{\operatorname{rank} V}
$$

for every proper subbundle $V^{\prime} \subset V$ such that $\Phi\left(V^{\prime}\right) \subset V^{\prime} \otimes K$. The Higgs bundle $(V, \Phi)$ is polystable if $(V, \Phi)=\oplus_{i}\left(V_{i}, \Phi_{i}\right)$ where $\left(V_{i}, \Phi_{i}\right)$ is a stable Higgs bundles and $\operatorname{deg} V_{i} / \operatorname{rank} V_{i}=\operatorname{deg} V / \operatorname{rank} V$.

If we consider $G=\operatorname{SL}(n, \mathbb{C})$ we have to add that $\operatorname{det}(V) \cong \mathcal{O}$ (hence $\operatorname{deg} V=0)$ and $\operatorname{Tr}(\Phi)=0$.

The notion of stability emerges from the study of the Hitchin equations. The equivalence between the existence of solutions to these equations and the $\alpha$-polystability of Higgs bundles is known as the Hitchin-Kobayashi correspondence, which we state below.

Theorem 2.10. Let $(E, \varphi)$ be a $G$-Higgs bundle over a Riemann surface $X$ with volume form $\omega$. Then $(E, \varphi)$ is $\alpha$-polystable if and only if there exists a reduction $h$ of the structure group of $E$ from $H^{\mathbb{C}}$ to $H$, that is a smooth section of $E\left(H^{\mathbb{C}} / H\right)$, such that

$$
F_{h}-\left[\varphi, \tau_{h}(\varphi)\right]=-i \alpha \omega
$$

where $\tau_{h}: \Omega^{1,0}\left(E\left(\mathfrak{m}^{\mathbb{C}}\right)\right) \rightarrow \Omega^{0,1}\left(E\left(\mathfrak{m}^{\mathbb{C}}\right)\right)$ is the combination of the anti-holomorphic involution in $E\left(\mathfrak{m}^{\mathbb{C}}\right)$ defined by the compact real form at each point determined by $h$ and the conjugation of 1-forms, and $F_{h}$ is the curvature of the unique $H$-connection compatible with the holomorphic structure of $E$ (the Chern connection).

This theorem was proved by Hitchin in the case of $\mathrm{SL}(2, \mathbb{C})$, by Simpson when $G$ is complex, and in [BGM03, GGM09] for a general reductive real Lie group $G$

Remark 2.11. There is a theorem similar to Theorem 2.10 for $L$-twisted $G$-Higgs bundles (see Remark 2.5 and [GGM09]) for an arbitrary line bundle $L$. If $(E, \varphi)$ is such a pair, one fixes a Hermitian metric $h_{L}$ on $L$, and looks for a reduction of structure group $h$ of $E$ from $H^{\mathbb{C}}$ to $H$ satisfying

$$
F_{h}-\left[\varphi, \tau_{h}(\varphi)\right] \omega=-i \alpha \omega
$$

where now $\tau_{h}: \Omega^{0}\left(E\left(\mathfrak{m}^{\mathbb{C}}\right) \otimes L\right) \rightarrow \Omega^{0}\left(E\left(\mathfrak{m}^{\mathbb{C}}\right) \otimes L\right)$ is the combination of the antiholomorphic involution in $E\left(\mathfrak{m}^{\mathbb{C}}\right)$ defined by the compact real form at each point determined by $h$ and the metric $h_{L}$. 
2.3. Higgs bundles and representations. Fix a base point $x \in X$. A representation of $\pi_{1}(X, x)$ in $G$ is a homomorphism $\pi_{1}(X, x) \longrightarrow G$. After fixing a presentation of $\pi_{1}(X, x)$, the set of all such homomorphisms, $\operatorname{Hom}\left(\pi_{1}(X, x), G\right)$, can be identified with the subset of $G^{2 g}$ consisting of $2 g$-tuples $\left(A_{1}, B_{1}, \cdots, A_{g}, B_{g}\right)$ satisfying the algebraic equation $\prod_{i=1}^{g}\left[A_{i}, B_{i}\right]=1$. This shows that $\operatorname{Hom}\left(\pi_{1}(X, x), G\right)$ is an algebraic variety.

The group $G$ acts on $\operatorname{Hom}\left(\pi_{1}(X, x), G\right)$ by conjugation:

$$
(g \cdot \rho)(\gamma)=g \rho(\gamma) g^{-1},
$$

where $g \in G, \rho \in \operatorname{Hom}\left(\pi_{1}(X, x), G\right)$ and $\gamma \in \pi_{1}(X, x)$. If we restrict the action to the subspace $\operatorname{Hom}^{+}\left(\pi_{1}(X, x), G\right)$ consisting of reductive representations, the orbit space is Hausdorff. We recall that a reductive representation is one whose composition with the adjoint representation in $\mathfrak{g}$ decomposes as a direct sum of irreducible representations. This is equivalent to the condition that the Zariski closure of the image of $\pi_{1}(X, x)$ in $G$ is a reductive group. Define the moduli space of representations of $\pi_{1}(X, x)$ in $G$ to be the orbit space

$$
\mathcal{R}(G)=\operatorname{Hom}^{+}\left(\pi_{1}(X, x), G\right) / G .
$$

This is a real algebraic variety. For another point $x^{\prime} \in X$, the fundamental groups $\pi_{1}(X, x)$ and $\pi_{1}\left(X, x^{\prime}\right)$ are identified by an isomorphism unique up to an inner automorphism. Consequently, $\mathcal{R}(G)$ is independent of the choice of the base point $x$.

Given a representation $\rho: \pi_{1}(X, x) \longrightarrow G$, there is an associated flat principal $G$ bundle on $X$, defined as

$$
E_{\rho}=\widetilde{X} \times{ }_{\rho} G,
$$

where $\widetilde{X} \longrightarrow X$ is the universal cover and $\pi_{1}(X, x)$ acts on $G$ via $\rho$. This gives in fact an identification between the set of equivalence classes of representations $\operatorname{Hom}\left(\pi_{1}(X), G\right) / G$ and the set of equivalence classes of flat principal $G$-bundles, which in turn is parametrized by the (non-abelian) cohomology set $H^{1}(X, G)$. We have the following.

Theorem 2.12. Let $G$ be a semisimple real Lie group. Then there is a homeomorphism $\mathcal{R}(G) \cong \mathcal{M}(G)$.

The proof of Theorem 2.12 is the combination of Theorem 2.10 and the following theorem of Corlette [Cor88], also proved by Donaldson [Don87] when $G=\operatorname{SL}(2, \mathbb{C})$.

Theorem 2.13. Let $\rho$ be a representation of $\pi_{1}(X)$ in $G$ with corresponding flat $G$ bundle $E_{\rho}$. Let $E_{\rho}(G / H)$ be the associated $G / H$-bundle. Then the existence of a harmonic section of $E_{\rho}(G / H)$ is equivalent to the reductiveness of $\rho$.

Remark 2.14. Theorem 2.12 can be extended to reductive groups replacing $\pi_{1}(X)$ by its universal central extension.

We can assign a topological invariant to a representation $\rho$ given by the characteristic class $c(\rho):=c\left(E_{\rho}\right) \in \pi_{1}(G)$ corresponding to $E_{\rho}$. To define this, let $\widetilde{G}$ be the universal covering group of $G$. We have an exact sequence

$$
1 \longrightarrow \pi_{1}(G) \longrightarrow \widetilde{G} \longrightarrow G \longrightarrow 1
$$


which gives rise to the (pointed sets) cohomology sequence

$$
H^{1}(X, \widetilde{G}) \longrightarrow H^{1}(X, G) \stackrel{c}{\longrightarrow} H^{2}\left(X, \pi_{1}(G)\right) .
$$

Since $\pi_{1}(G)$ is abelian, we have

$$
H^{2}\left(X, \pi_{1}(G)\right) \cong \pi_{1}(G),
$$

and $c\left(E_{\rho}\right)$ is defined as the image of $E$ under the last map in (2.5). Thus the class $c\left(E_{\rho}\right)$ measures the obstruction to lifting $E_{\rho}$ to a flat $\widetilde{G}$-bundle, and hence to lifting $\rho$ to a representation of $\pi_{1}(X)$ in $\widetilde{G}$. For a fixed $c \in \pi_{1}(G)$, we can consider the subvariety of the moduli space of representations $\mathcal{R}_{c}(G)$ defined by

$$
\mathcal{R}_{c}(G):=\{\rho \in \mathcal{R}(G): c(\rho)=c\} .
$$

Similarly, we can assign a topological invariant to a $G$-Higgs bundle $(E, \varphi)$. Assuming that $G$ is connected (and hence $H$ is connected), topologically, $H^{\mathbb{C}}$-bundles $E$ on $X$ are classified by a characteristic class $c=c(E) \in \pi_{1}\left(H^{\mathbb{C}}\right)=\pi_{1}(H)=\pi_{1}(G)$. Again, this comes from considering the exact sequence

$$
1 \longrightarrow \pi_{1}\left(H^{\mathbb{C}}\right) \longrightarrow \widetilde{H^{\mathbb{C}}} \longrightarrow H^{\mathbb{C}} \longrightarrow 1
$$

and the corresponding (pointed sets) cohomology sequence

$$
H^{1}\left(X, \underline{\widetilde{H}^{\mathbb{C}}}\right) \longrightarrow H^{1}\left(X, \underline{H^{\mathbb{C}}}\right) \stackrel{c}{\longrightarrow} H^{2}\left(X, \pi_{1}\left(H^{\mathbb{C}}\right)\right) .
$$

For a fixed such class $c$, the subvariety $\mathcal{M}_{c}(G) \subset \mathcal{M}(G)$ is defined as the set of isomorphism classes of polystable $G$-Higgs bundles $(E, \varphi)$ such that $c(E)=c$.

If $G$ is semisimple, of course, the homeomorphism in Theorem 2.12 restricts to give a homeomorphism

$$
\mathcal{R}_{c}(G) \cong \mathcal{M}_{c}(G) .
$$

Proposition 2.15. Let $G$ be a connected semisimple real Lie group. Then

(1) There is a homomorphism

$$
\pi_{0}(\mathcal{R}(G)) \cong \pi_{0}(\mathcal{M}(G)) \longrightarrow \pi_{1}(G) .
$$

(2) If $G$ is compact or complex, the homomorphism in (1) is an isomorphism.

The proof of (2) in Proposition 2.15 is due J. Li [Li93]. An alternative proof using Higgs bundles, and including the case of non-connected reductive Lie groups is given in [GO14].

The homomorphism in (1) of Proposition 2.15 for a real form of a complex semisimple Lie group may be neither surjective nor injective, as we will see in Section 3.

3. A Basic exAmple: $G=\operatorname{SL}(2, \mathbb{R})$

We consider $G=\operatorname{SL}(2, \mathbb{R})$. Let $X$ be a compact Riemann surface of genus $g \geq 2$. Given a representation $\rho: \pi_{1}(X) \rightarrow \mathrm{SL}(2, \mathbb{R})$, the invariant associated to $\rho$, defined in Section 2.3 is an integer $d \in \pi_{1}(\mathrm{SL}(2, \mathbb{R}) \cong \mathbb{Z}$. This is the Euler class $d \in \mathbb{Z}$ of the corresponding flat $\operatorname{SL}(2, \mathbb{R})$-bundle $E_{\rho}$. We can then define the subvarieties

$$
\mathcal{R}_{d}:=\{\rho \in \mathcal{R}(\mathrm{SL}(2, \mathbb{R})) \text { : with Euler class } d\} .
$$


By Milnor [Mil58], $\mathcal{R}_{d}$ is empty unless

$$
|d| \leq g-1 .
$$

A maximal compact subgroup of $\mathrm{SL}(2, \mathbb{R})$ can be identified with $\mathrm{U}(1)$, and its complexification $\mathbb{C}^{*}$ can be embedded in $\operatorname{SL}(2, \mathbb{C})$ as the matrices of the form

$$
\left(\begin{array}{cc}
a & 0 \\
0 & a^{-1}
\end{array}\right),
$$

with $a \in \mathbb{C}^{*}$. On the other hand, in this case $\mathfrak{m}^{\mathbb{C}}$ coincides with the matrices

$$
\left(\begin{array}{ll}
0 & b \\
c & 0
\end{array}\right),
$$

with $b, c \in \mathbb{C}$.

An $\mathrm{SL}(2, \mathbb{R})$-Higgs bundle is then simply a triple $(L, \beta, \gamma)$, where $L$ is a holomorphic line bundle over $X$ and $\beta \in H^{0}\left(X, L^{2} K\right)$ and $\gamma \in H^{0}\left(X, L^{-2} K\right)$. It can also be viewed as the $\mathrm{SL}(2, \mathbb{C})$-Higgs bundle $(V, \Phi)$ obtained by extension of structure group given by $V=L \oplus L^{-1}$, and

$$
\Phi=\left(\begin{array}{ll}
0 & \beta \\
\gamma & 0
\end{array}\right) .
$$

The topological invariant attached to the Higgs bundle $(L, \beta, \gamma)$ is then $\operatorname{deg} L$, the degree of $L$ (its first Chern class). As for representations, we can define the subvariety $\mathcal{M}_{d} \subset \mathcal{M}(\mathrm{SL}(2, \mathbb{R}))$ as the $\operatorname{SL}(2, \mathbb{R})$-Higgs bundles with fixed $\operatorname{deg} L=d$. Here the natural gauge transformations are $\mathbb{C}^{*}$-transformations, that is, those of $L$. Allowing $\mathrm{SL}(2, \mathbb{C})$-transformation naturally identifies $\mathcal{M}_{d}$ and $\mathcal{M}_{-d}$ inside $\mathcal{M}(\mathrm{SL}(2, \mathbb{C}))$. Now, the semistability condition gives a constraint on the possible degrees that $L$ may have, namely, we must have

$$
|d| \leq g-1
$$

This can be seen very easily ([Hit87]). Assume that $d \geq 0$ (similar argument for $d \leq 0$ ). Suppose that $d>g-1$. Then $\gamma=0$ and $L$ is a $\Phi$-invariant line subbundle of $V$. By the semistability of $(V, \Phi)$ we must have that $d \leq 0$ which gives a contradiction. Considering the homeomorphism $\mathcal{R}_{d} \cong \mathcal{M}_{d}$, this gives a Higgs bundle proof of the Milnor inequality [Mil58].

The moduli space of representations of $\pi_{1}(X)$ in $\mathrm{SL}(2, \mathbb{R})$ was studied by Goldman [Gol88], who showed that for $d$ satisfying $d=g-1$ (same for $d=1-g$ ) there are $2^{2 g}$ isomorphic connected components that can be identified with Teichmüller space, and showed that for $d$ such that $|d|<g-1$ there is only one connected component. This was also proved by Hitchin [Hit87], who also gave a very explicit description of each component. Namely, for $0<|d|<g-1, \mathcal{M}_{d}$ is the total space of a holomorphic complex vector bundle of rank $g+2|d|-1$ over a $2^{2 g}$-fold covering of the $2 g-2-2|d|$-symmetric power of $X$

$$
\operatorname{Sym}^{2 g-2-2|d|} X \text {. }
$$


To see this, assume that $d>0$ (the case $d<0$ is similar), then the pair $(L, \gamma)$ defines an element in $\operatorname{Sym}^{2 g-2-2 d}(X)$, given by the zeros of $\gamma$. But if $L_{0}$ is a line bundle of degree 0 of order two, that is, $L_{0}^{2}=\mathcal{O}$, then the element $\left(L L_{0}, \gamma\right)$ defines also the same divisor in $\operatorname{Sym}^{2 g-2-2 d}(X)$. Hence the set of pairs $(L, \gamma)$ gives a point in the $2^{2 g}$-fold covering of $\operatorname{Sym}^{2 g-2-2 d}(X)$. The section $\beta$ now gives the fibre of the vector bundle. Notice Riemann-Roch implies that $H^{1}\left(X, L^{2} K\right)=0$. The case of $d=0$ is a bit more involved (see [Hit87] and [GR18])

From this we conclude the following.

Proposition 3.1. (1) $\operatorname{dim} \mathcal{M}_{d}=3 g-3$,

(2) $\mathcal{M}_{d}$ is connected if $|d|<g-1$,

(3) $\mathcal{M}_{d}$ has $2^{2 g}$ connected components if $|d|=g-1$, each isomorphic to $\mathbb{C}^{3 g-3}$ (the fibre of a rank $3 g-3$ vector bundle over a $2^{2 g}$-fold covering of a point!).

(3) is clear since if $\operatorname{deg} L=g-1$, the line bundle $L^{-2} \otimes K$ is of zero degree and hence has a section (unique up to multiplicaton by a scalar) if and only if $L^{-2} \otimes K \cong \mathcal{O}$, i.e. if $L$ is a square root of $K$. For each of the $2^{2 g}$ choices of square root $L=K^{1 / 2}$, one has a connected component which is parametrized by $\beta \in H^{0}\left(X, K^{2}\right)$. Each of these components is diffeomorphic to Teichmüller space.

\section{The Hitchin map, split Real Forms and Hitchin components}

For details on this section, see [GPR18].

4.1. Maximal split subgroup and Chevalley map. Let $\mathfrak{g}$ be a reductive real Lie algebra with a Cartan involution $\theta$ decomposing $\mathfrak{g}$ as $\mathfrak{g}=\mathfrak{h} \oplus \mathfrak{m}$. Given a maximal subalgebra $\mathfrak{a} \subset \mathfrak{m}$ it follows that it must be abelian, and one can easily prove that its elements are semisimple and diagonalizable over the real numbers (cf. [Kna96, Chap.VI], note that Knapp proves it for semisimple Lie algebras, but for reductive Lie algebras it suffices to use invariance of the centre and the semisimple part of $[\mathfrak{g}, \mathfrak{g}])$ under the Cartan involution. Any such subalgebra is called a maximal anisotropic Cartan subalgebra of $\mathfrak{g}$. By extension, its complexification $\mathfrak{a}^{\mathbb{C}}$ is called a maximal anisotropic Cartan subalgebra of $\mathfrak{g}^{\mathbb{C}}$ (with respect to $\mathfrak{g}$ ). A maximal anisotropic Cartan subalgebra $\mathfrak{a}$ can be completed to a $\theta$-equivariant Cartan subalgebra of $\mathfrak{g}$, namely, a subalgebra whose complexification is a Cartan subalgebra of $\mathfrak{g}^{\mathbb{C}}$. Indeed, define

$$
\mathfrak{d}=\mathfrak{t} \oplus \mathfrak{a}
$$

where $\mathfrak{t} \subset \mathfrak{c}_{\mathfrak{h}}(\mathfrak{a}):=\{x \in \mathfrak{h}:[x, \mathfrak{a}]=0\}$ is a maximal abelian subalgebra ([Kna96], Proposition 6.47). Cartan subalgebras of this kind (and their complexifications) are called maximally split.

The dimension of maximal anisotropic Cartan subalgebras of a real reductive Lie algebra $\mathfrak{g}$ is called the the real (or split) rank of $\mathfrak{g}$. This number measures the degree of compactness of real forms: indeed, a real form is compact (that is, its adjoint group is compact) if and only if $\operatorname{rk}_{\mathbb{R}}(\mathfrak{g})=0$. On the other hand, a real form is defined to be split if $\operatorname{rk}_{\mathbb{R}}(\mathfrak{g})=\mathrm{rkg}^{\mathbb{C}}$. Note that the split rank depends on the involution $\theta$ associated with the real form, when $\mathfrak{g}$ is not semisimple. 
In [KR71], Kostant and Rallis give a procedure to construct a $\theta$-invariant subalgebra $\widehat{\mathfrak{g}} \subset \mathfrak{g}$ such $\widehat{\mathfrak{g}} \subset(\widehat{\mathfrak{g}})^{\mathbb{C}}$ is a split real form, whose Cartan subalgebra is $\mathfrak{a}$ and such that $\mathfrak{z}(\widehat{\mathfrak{g}})=\mathfrak{z}(\mathfrak{g}) \cap \mathfrak{m}$. Their construction relies on the following notion. A three dimensional subalgebra $(\mathrm{TDS}) \mathfrak{s}^{\mathbb{C}} \subset \mathfrak{g}^{\mathbb{C}}$ is the image of an injective morphism $\mathfrak{s l}(2, \mathbb{C}) \rightarrow \mathfrak{g}^{\mathbb{C}}$. A TDS is called normal if $\operatorname{dim} \mathfrak{s}^{\mathbb{C}} \cap \mathfrak{h}^{\mathbb{C}}=1$ and $\operatorname{dim} \mathfrak{s}^{\mathbb{C}} \cap \mathfrak{m}^{\mathbb{C}}=2$. It is called principal if it is generated by elements $\{e, f, x\}$, where $e$ and $f$ are nilpotent regular elements in $\mathfrak{m}^{\mathbb{C}}$, and $x \in \mathfrak{h}^{\mathbb{C}}$ is semisimple. A set of generators satisfying such relations is called a normal triple.

A subalgebra $\hat{\mathfrak{g}} \subset \mathfrak{g}$ generated by $\mathfrak{a}$ and $\mathfrak{s}^{\mathbb{C}} \cap \mathfrak{g}$, where $\mathfrak{s}^{\mathbb{C}}$ is a principal normal TDS invariant by the involution defining $\mathfrak{g}$ inside of $\mathfrak{g}^{\mathbb{C}}$ is called a maximal split subalgebra.

Remark 4.1. Let $\mathfrak{g}^{\mathbb{C}}$ be a complex reductive Lie algebra, and let $\left(\mathfrak{g}^{\mathbb{C}}\right)_{\mathbb{R}}$ be its underlying real reductive algebra. Then, the maximal split subalgebra of $\left(\mathfrak{g}^{\mathbb{C}}\right)_{\mathbb{R}}$ is isomorphic to the split real form $\mathfrak{g}_{\text {split }}$ of $\mathfrak{g}^{\mathbb{C}}$. It is clearly split within its complexification and it is maximal within $\left(\mathfrak{g}^{\mathbb{C}}\right)_{\mathbb{R}}$ with this property, which can be easily checked by identifying $\left(\mathfrak{g}^{\mathbb{C}}\right)_{\mathbb{R}} \cong \mathfrak{g}_{\text {split }} \oplus i \mathfrak{g}_{\text {split }}$.

We say that a real Lie group is split if its Lie algebra is split. Let $(G, H, \theta, B)$ be a real reductive Lie group. The tuple $(\widehat{G}, \widehat{H}, \widehat{\theta}, \widehat{B})$, where $\widehat{G} \subset G$ is the analytic subgroup correspoding to the maximal split subalgebra $\widehat{\mathfrak{g}} \subset \mathfrak{g}, \widehat{H}:=\exp (i \widehat{\mathfrak{h}}) \leq H$, and $\widehat{\theta}$ and $\widehat{B}$ are obtained by restriction, is a reductive Lie group to which we refer as the maximal split subgroup of $(G, H, \theta, B)$.

The restriction to $\mathfrak{a}$ of the adjoint representation of $\mathfrak{g}$ yields a decomposition of $\mathfrak{g}$ into a-eigenspaces

$$
\mathfrak{g}=\bigoplus_{\lambda \in \Lambda(\mathfrak{a})} \mathfrak{g}_{\lambda}
$$

where $\Lambda(\mathfrak{a}) \subset \mathfrak{a}^{*}$ is called the set of restricted roots of $\mathfrak{g}$ with respect to $\mathfrak{a}$. The set $\Lambda(\mathfrak{a})$ forms a root system (see [Kna96, Chap. II, Sec. 5]), which may not be reduced (that is, there may be roots whose double is also a root). The name restricted roots is due to the following fact: extending restricted roots by $\mathbb{C}$-linearity, we obtain $\Lambda\left(\mathfrak{a}^{\mathbb{C}}\right) \subset\left(\mathfrak{a}^{\mathbb{C}}\right)^{*}$, also called restricted roots.

We define the restricted Weyl group of $\mathfrak{g}^{\mathbb{C}}$ associated to $\mathfrak{a}^{\mathbb{C}}, W\left(\mathfrak{a}^{\mathbb{C}}\right)$, to be the group of automorphisms of $\mathfrak{a}^{\mathbb{C}}$ generated by reflections on the hyperplanes defined by the restricted roots $\lambda \in \Lambda\left(\mathfrak{a}^{\mathbb{C}}\right)$.

Proposition 4.2. Let $(G, H, \theta, B)$ be a real reductive Lie group and $(\widehat{G}, \widehat{H}, \widehat{\theta}, \widehat{B})<$ $(G, H, \theta, B)$ be its maximal split subgroup. Let $a=\operatorname{dim} \mathfrak{a}^{\mathbb{C}}$. Then:

(1) Restriction induces an isomorphism

$$
\mathbb{C}\left[\mathfrak{m}^{\mathbb{C}}\right]^{H^{\mathbb{C}}} \cong \mathbb{C}\left[\mathfrak{a}^{\mathbb{C}}\right]^{W\left(\mathfrak{a}^{\mathbb{C}}\right)} \cong \mathbb{C}\left[\widehat{\mathfrak{m}}^{\mathbb{C}}\right]^{\widehat{H}^{\mathbb{C}}} .
$$

(2) $\mathbb{C}\left[\mathfrak{a}^{\mathbb{C}}\right]^{W\left(\mathfrak{a}^{\mathbb{C}}\right)}$ is generated by homogeneous polynomials of degrees $d_{1}, \ldots, d_{a}$, canonically determined by $(G, \theta)$. 
(3) The degrees of the homogeneous polynomials for $(G, H, \theta, B)$ and $(\widehat{G}, \widehat{H}, \widehat{\theta}, \widehat{B})$ coincide

We thus have an algebraic morphism, called the Chevalley map

$$
\chi: \mathfrak{m}^{\mathbb{C}} \rightarrow \mathfrak{m}^{\mathbb{C}} / / H^{\mathbb{C}} \cong \mathfrak{a}^{\mathbb{C}} / W\left(\mathfrak{a}^{\mathbb{C}}\right)
$$

where the double quotient sign // stands for the affine GIT quotient.

4.2. The Hitchin map. Let $(G, H, \theta, B)$ be a reductive real Lie group. Consider the Chevalley morphism (4.1):

$$
\chi: \mathfrak{m}^{\mathbb{C}} \rightarrow \mathfrak{a}^{\mathbb{C}} / W\left(\mathfrak{a}^{\mathbb{C}}\right) .
$$

This map is $\mathbb{C}^{\times}$-equivariant. In particular, it induces a morphism

$$
h: \mathfrak{m}^{\mathbb{C}} \otimes K \rightarrow \mathfrak{a}^{\mathbb{C}} \otimes K / W\left(\mathfrak{a}^{\mathbb{C}}\right) .
$$

The map $\chi$ is also $H^{\mathbb{C}}$-equivariant, thus defining a morphism

$$
h: \mathcal{M}(G) \rightarrow B(G):=H^{0}\left(X, \mathfrak{a}^{\mathbb{C}} \otimes K / W\left(\mathfrak{a}^{\mathbb{C}}\right)\right) .
$$

The map $h$ is called the Hitchin map, and the space $B(G)$ is called the Hitchin base.

In more concrete terms Let $p$ be an $H^{\mathbb{C}}$-invariant homogeneous polynomial of degree $d$ on $\mathfrak{m}^{\mathbb{C}}$. Then, if $(E, \varphi)$ is a $G$-Higgs bundle, the evaluation of $p$ on $\varphi$ gives an element of $H^{0}\left(X, K^{d}\right)$, so $p$ induces a map

$$
p: \mathcal{M}(G) \longrightarrow H^{0}\left(X, K^{d}\right) .
$$

Let $p_{1}, \ldots, p_{r}$ with $r=\operatorname{rk}(G)$ be a basis of the ring of $H^{\mathbb{C}}$-invariant homogeneous polynomials on $\mathfrak{m}^{\mathbb{C}}$. This basis defines a map

$$
\mathcal{M}(G) \rightarrow \bigoplus_{i=1}^{r} H^{0}\left(X, K^{d_{i}}\right)
$$

where, for $i=1,2, \ldots, r, d_{i}$ is the degree of $p_{i}$. These degrees are independent of the bases and they only depend on $G$. This is the Hitchin map and hence the Hitchin base is

$$
B(G)=\bigoplus_{i=1}^{r} H^{0}\left(X, K^{d_{i}}\right)
$$

If $G$ is complex and $E$ is a stable $G$-bundle, one has (see [Hit87b]) that

$$
\operatorname{dim} B(G)=\operatorname{dim} H^{0}(X, E(\mathfrak{g}) \otimes K)
$$

and hence the dimension of $B(G)$ coincides with the dimension of the moduli space of $G$-bundles, which is half the dimension of $\mathcal{M}(G)$. In [Hit87b] Hitchin shows that $\mathcal{M}(G)$ is a completely algebraically integrable system and that, for a classical group, the generic fibre is either a Jacobian or a Prym variety of a curve covering $X$, which is called the spectral curve. The description of the fibres for a general complex reductive Lie group is given by Donagi and Gaitsgory [DG01] in terms of a cameral cover of the curve. The description of the fibration for general real forms is not yet fully understood (for some partial results see [Peo13, Sch13, HS14, GP19]). 
4.3. Hitchin components for $\mathrm{SL}(n, \mathbb{R})$-Higgs bundles. We will illustrate first how Hitchin constructed in [Hit92] special components of the moduli space of SL $(n, \mathbb{R})$-Higgs bundles as the image of certain sections of the Hitchin map. To explain this, recall that Cartan decomposition of $\mathfrak{s l}(n, \mathbb{R})$ is given by

$$
\mathfrak{s l}(n, \mathbb{R})=\mathfrak{s o}(n)+\mathfrak{m},
$$

where $\mathfrak{m}=\{$ symmetric real matrices of trace 0$\}$. A SL $(n, \mathbb{R})$ - Higgs bundle is thus a pair $(E, \varphi)$, where $E$ is a principal holomorphic $\mathrm{SO}(n, \mathbb{C})$-bundle over $X$ and the Higgs field is a holomorphic section

$$
\varphi \in H^{0}\left(E\left(\mathfrak{m}^{\mathbb{C}}\right) \otimes K\right) .
$$

Using the standard representations of $\mathrm{SO}(n, \mathbb{C})$ in $\mathbb{C}^{n}$ one can associate to $E$ a holomorphic vector bundle $V$ of rank $n$ with $\operatorname{det} V=\mathcal{O}$ together with a nondegenerate quadratic form $Q \in H^{0}\left(S^{2} V^{*}\right)$.

A SL $(n, \mathbb{R})$-Higgs bundle is then in correspondence with a triple

$$
(V, Q, \varphi),
$$

where the Higgs field is a symmetric and traceless endomorphism $\varphi: V \rightarrow V \otimes K$.

The simplest case is to consider the complex Lie group $\mathrm{SL}(2, \mathbb{C})$ and its split real form $\mathrm{SL}(2, \mathbb{R})$. The Lie algebra $\mathfrak{s l}(2, \mathbb{C})$ has rank 1 and the algebra of invariant polynomials on it is generated by $p_{2}$ of degree 2 obtained from the characteristic polynomial

$$
\operatorname{det}(x 1-A)=x^{2}+p_{2}(A)
$$

of a trace-free matrix. We are going to define a section of the Hitchin map

$$
\begin{aligned}
p: \mathcal{M} & \rightarrow H^{0}\left(K^{2}\right) \\
(E, \varphi) & \mapsto p_{2}(\varphi)
\end{aligned}
$$

where here $\mathcal{M}$ denotes the moduli space of polystable $\mathrm{SL}(2, \mathbb{C})$-Higgs bundles. This section will give an isomorphism between the vector space $H^{0}\left(K^{2}\right)$ and a connected component of the moduli space $\mathcal{M}(\mathrm{SL}(2, \mathbb{R})) \subset \mathcal{M}$ of polystable $\mathrm{SL}(2, \mathbb{R})$-Higgs bundles. To construct the section, we consider the elements

$$
\left\langle x=\left(\begin{array}{cc}
1 & 0 \\
0 & -1
\end{array}\right), e=\left(\begin{array}{ll}
0 & 1 \\
0 & 0
\end{array}\right), \tilde{e}=\left(\begin{array}{ll}
0 & 0 \\
1 & 0
\end{array}\right)\right\rangle \cong \mathfrak{s l}(2, \mathbb{C})
$$

that satisfy

$$
[x, e]=2 e,[x, \tilde{e}]=-2 \tilde{e} \text { and }[e, \tilde{e}]=x,
$$

where $x$ is an element of the Cartan subalgebra (a semisimple element) and $e, \tilde{e}$ are nilpotent. The pair $\left(K^{1 / 2} \oplus K^{-1 / 2}, \varphi=\tilde{e}-\alpha e=\left(\begin{array}{cc}0 & -\alpha \\ 1 & 0\end{array}\right)\right)$, where $\alpha \in H^{0}\left(K^{2}\right)$, is a $\mathrm{SL}(2, \mathbb{R})$-Higgs bundle. In the vector bundle $K^{1 / 2} \oplus K^{-1 / 2}$ we have the orthogonal structure $Q=\left(\begin{array}{ll}0 & 1 \\ 1 & 0\end{array}\right)$ and the Higgs field is symmetric with respect to this orthogonal 
form. The section is finally defined by

$$
s(\alpha)=\left(K^{1 / 2} \oplus K^{-1 / 2}, \varphi=\left(\begin{array}{cc}
0 & -\alpha \\
1 & 0
\end{array}\right)\right) .
$$

That is, the pairs

$$
\left\{\left(K^{1 / 2} \oplus K^{-1 / 2}, \varphi=\left(\begin{array}{cc}
0 & -\alpha \\
1 & 0
\end{array}\right)\right)\right\}_{\alpha \in H^{0}\left(K^{2}\right)}
$$

form a connected component of $\mathcal{M}(\mathrm{SL}(2, \mathbb{R}))$ of dimension $6 g-6$, and there are $2^{2 g}$ connected components isomorphic to this one - the number of possible choices of the square $K^{1 / 2}$. These are precisely the components with maximal integer invariant given by the Milnor inequality, described in Section 3.

Now consider the general case $\operatorname{SL}(n, \mathbb{R})$ which is the split real form of $\operatorname{SL}(n, \mathbb{C})$. The Lie algebra $\mathfrak{s l}(n, \mathbb{C})$ has rank $n-1$ and a basis for the invariant polynomials on $\mathfrak{s l}(n, \mathbb{C})$ is provided by the coefficients of the characteristic polynomial of a trace-free matrix,

$$
\operatorname{det}(x-A)=x^{n}+p_{1}(A) x^{n-2}+\ldots+p_{n-1}(A),
$$

where $\operatorname{deg}\left(p_{i}\right)=i+1$. Ehe Hitchin map

$$
h: \mathcal{M}(\mathrm{SL}(n, \mathbb{C})) \rightarrow \bigoplus_{i=1}^{n-1} H^{0}\left(K^{i+1}\right)
$$

is defined by

$$
h(E, \varphi)=\left(p_{1}(\varphi), \ldots, p_{n-1}(\varphi)\right),
$$

where $\mathcal{M}(\mathrm{SL}(n, \mathbb{C}))$ is the moduli space of polystable $\mathrm{SL}(n, \mathbb{C})$-Higgs bundles.

We are going to define a section of this map that will give an isomorphism between the vector space $\bigoplus_{i=1}^{n-1} H^{0}\left(K^{i+1}\right)$ and a connected component of the moduli space $\mathcal{M}(\mathrm{SL}(n, \mathbb{R})) \subset \mathcal{M}(\mathrm{SL}(n, \mathbb{C}))$ of polystable $\mathrm{SL}(n, \mathbb{R})$-Higgs bundles.

A nilpotent element $e \in \mathfrak{s l}(n, \mathbb{C})$ is called regular if its centralizer is $(n-1)$-dimensional. In $\mathfrak{s l}(n, \mathbb{C})$ a regular nilpotent element is conjugate to an element

$$
e=\sum_{\alpha \in \Pi} X_{\alpha}
$$

where $\Pi=\left\{\alpha_{i}=e_{i}-e_{i+1}, 1 \leq i \leq n-1\right\}$ and $X_{\alpha_{i}}=E_{i, i+1}$ is a root vector for $\alpha_{i}$, that is,

$$
e=\left(\begin{array}{cccccc}
0 & 1 & 0 & \cdots & \cdots & 0 \\
\vdots & 0 & 1 & 0 & \cdots & 0 \\
\vdots & \vdots & & \ddots & & \vdots \\
\vdots & \vdots & & & \ddots & 0 \\
\vdots & \vdots & & & & 1 \\
0 & 0 & \cdots & \cdots & \cdots & 0
\end{array}\right)
$$


Any nilpotent element can be embedded in a 3-dimensional simple subalgebra $\langle x, e, \tilde{e}\rangle \cong$ $\mathfrak{s l}(2, \mathbb{C})$, where $x$ is semisimple, $e$ and $\tilde{e}$ are nilpotent, and they satisfy

$$
[x, e]=2 e ; \quad[x, \tilde{e}]=-2 \tilde{e} ; \quad[e, \tilde{e}]=x .
$$

The adjoint action

$$
\langle x, e, \tilde{e}\rangle \cong \mathfrak{s l}(2, \mathbb{C}) \rightarrow \operatorname{End}(\mathfrak{s l}(n, \mathbb{C}))
$$

of this subalgebra breaks up the Lie algebra $\mathfrak{s l}(n, \mathbb{C})$ as a direct sum of irreducible representations

$$
\mathfrak{s l}(n, \mathbb{C})=\bigoplus_{i=1}^{n-1} V_{i}
$$

with $\operatorname{dim}\left(V_{i}\right)=2 i+1$. That is, each $V_{i}$ is the irreducible representation $S^{2 i} \mathbb{C}^{2}$, where $\mathbb{C}^{2}$ is the standard representation of $\mathfrak{s l}(2, \mathbb{C})$, and the eigenvalues of ad $x$ on $V_{i}$ are $-2 i,-2 i+2, \ldots, 2 i-2,2 i$.

The highest weight vector of $V_{i}$, defined as a vector $e_{i} \in V_{i}$ that is an eigenvector for the action of $x$ and is in the kernel of $\operatorname{ad}(e)$, has eigenvalue $2 i$ for $\operatorname{ad} x$. We take $V_{1}=\langle x, e, \tilde{e}\rangle$ and $e=e_{1}$.

Given $\left(\alpha_{1}, \ldots, \alpha_{n-1}\right) \in \bigoplus_{i=1}^{n-1} H^{0}\left(K^{i+1}\right)$, we define the Higgs field in the Hitchin component by

$$
\varphi=\tilde{e}_{1}+\alpha_{1} e_{1}+\ldots+\alpha_{n-1} e_{n-1}
$$

and the vector bundle is given by

$$
S^{n-1}\left(K^{-1 / 2} \oplus K^{1 / 2}\right)=K^{-(n-1) / 2} \oplus K^{-(n-3) / 2} \oplus \cdots \oplus K^{(n-3) / 2} \oplus K^{(n-1) / 2} .
$$

The field $\varphi$ is given in the following order of the basis, $K^{(n-1) / 2} \oplus K^{(n-3) / 2} \oplus \cdots \oplus$ $K^{-(n-3) / 2} \oplus K^{-(n-1) / 2}$.

Hitchin [Hit92] generalized this construction to the split real form of any complex semisimple Lie group, proving the following.

Theorem 4.3. Let $S$ be a compact oriented surface of genus $g>1$. Let $G^{\mathbb{C}}$ be a complex semisimple Lie group and let $G$ be the split real form of $G^{\mathbb{C}}$. Then the moduli space $\mathcal{R}(G)$ of representations of the fundamental group of $S$ in $G$ has a connected component homeomorphic to a Euclidean space of dimension $\operatorname{dim} G(2 g-2)$. Moreover if $G^{\mathbb{C}}$ is of adjoint type this component is unique.

When $G=\operatorname{PSL}(2, \mathbb{R})$, this component can be identified with Teichmüller space, and this is why Hitchin calls these generalized Teichmüller components. They are now referred as Hitchin components.

4.4. The Hitchin-Kostant-Rallis section. Hitchin's construction of a section of the Hitchin map for the moduli space Higgs bundles for a complex group can be generalized to the moduli space of $G$-Higgs bundles for any real reductive Lie group [GPR18]. This construction relies on the Kostant-Rallis construction of a maximal split subalgebra explained in Section 4.1.

An element $x \in \mathfrak{m}^{\mathbb{C}}$ is said to be regular if $\operatorname{dim} \mathfrak{c}_{\mathfrak{m}} \mathbb{C}(x)=\operatorname{dim} \mathfrak{a}^{\mathbb{C}}$, where $\mathfrak{c}_{\mathfrak{m}} \mathbb{C}(x)=$ $\left\{y \in \mathfrak{m}^{\mathbb{C}}:[y, x]=0\right\}$. Denote the subset of regular elements of $\mathfrak{m}^{\mathbb{C}}$ by $\mathfrak{m}_{\text {reg }}^{\mathbb{C}}$. Regular 
elements are those whose $H^{\mathbb{C}}$-orbits are maximal dimensional, so this notion generalises the classical notion of regularity of an element of a complex reductive Lie algebra. Note that the intersection $\mathfrak{m}^{\mathbb{C}} \cap \mathfrak{g}_{\text {reg }}^{\mathbb{C}}$ is either empty or the whole of $\mathfrak{m}_{\text {reg }}^{\mathbb{C}}$. Here $\mathfrak{g}_{\text {reg }}^{\mathbb{C}}$ denotes the set of elements of $\mathfrak{g}^{\mathbb{C}}$ with maximal dimensional $G^{\mathbb{C}}$-orbit.

A real form $\mathfrak{g} \subset \mathfrak{g}^{\mathbb{C}}$ is called quasi-split if $\mathfrak{m}^{\mathbb{C}} \cap \mathfrak{g}_{\text {reg }}^{\mathbb{C}}=\mathfrak{m}_{\text {reg. }}^{\mathbb{C}}$. These include split real forms, and the Lie algebras $\mathfrak{s u}(p, p), \mathfrak{s u}(p, p+1), \mathfrak{s o}(p, p+2)$, and $\mathfrak{e}_{6(2)}$. Quasi-split real forms admit several equivalent characterizations: $\mathfrak{g}$ is quasi-split if and only if $\mathfrak{c}_{\mathfrak{g}}(\mathfrak{a})$ is abelian — which holds if and only if $\mathfrak{g}^{\mathbb{C}}$ contains a $\theta$-invariant Borel subalgebra — and if and only if $\mathfrak{m}^{\mathbb{C}} \cap \mathfrak{g}_{\text {reg }}^{\mathbb{C}}=\mathfrak{m}_{\text {reg }}$.

Consider the group $Q$, satisfying $\left(\operatorname{Ad}(G)^{\mathbb{C}}\right)^{\theta}=Q \operatorname{Ad}\left(H^{\mathbb{C}}\right)$. It is a finite group whose cardinality we denote by $N$. The following theorem is proved in [GPR18].

Theorem 4.4. Let $(G, H, \theta, B)$ be a reductive real Lie group, and let $(\widehat{G}, \widehat{H}, \widehat{\theta}, \widehat{B})$ be its maximal split subgroup. Then, the choice of a square root of $K$ determines $N$ inequivalent sections of the map

$$
h: \mathcal{M}(G) \rightarrow B(G)
$$

Each such section $s_{G}$ satisfies

1. If $G$ is quasi-split, $s_{G}(B(G))$ is contained in the stable locus of $\mathcal{M}(G)$, and in the smooth locus if $Z(G)=Z_{G}(\mathfrak{g})$.

2. If $G$ is not quasi-split, the image of the section is contained in the strictly polystable locus.

3. For arbitrary groups, the Higgs field is everywhere regular.

4. The section factors through $\mathcal{M}(\widehat{G})$.

5. If $G_{\text {split }}<G^{\mathbb{C}}$ is the split real form satisfying $\mathfrak{z}\left(\mathfrak{g}^{\mathbb{C}}\right) \cap i \mathfrak{u} \subset \mathfrak{m}, s_{G}$ is the factorization of the Hitchin section through $\mathcal{M}\left(G_{\text {split }}\right)$.

\section{Hermitian groups and maximal Toledo components}

In this section we will assume that $G$ is a connected, non-compact real simple Lie group of Hermitian type with finite centre. We fix a maximal compact subgroup $H \subset G$, with Cartan decomposition $\mathfrak{g}=\mathfrak{h}+\mathfrak{m}$. The centre $Z(H)$ of $H$ is isomorphic to $\mathrm{U}(1)$. For details on this section see [BGR17].

5.1. Hermitian symmetric spaces and Cayley transform. The homogenous space $G / H$ is an irreducible Hermitian symmetric space of non-compact type. The same symmetric space $G / H$ is given by all the finite coverings of the $\operatorname{group} \operatorname{Ad}(G)=G / Z(G)$, where $Z(G)$ is the centre of $G$. The vector subspace $\mathfrak{m}$ is isomorphic to the tangent space $T_{o} G / H$ at the point $o=e H$. Let $H^{\mathbb{C}}, \mathfrak{h}^{\mathbb{C}}$ and $\mathfrak{m}^{\mathbb{C}}$ be the complexifications of $H, \mathfrak{h}$ and $\mathfrak{m}$ respectively. The almost complex structure $J_{0}$ on $\mathfrak{m}=T_{o}(G / H)$, where $o \in G / H$ corresponds to the coset $H$, is induced by the adjoint action of an element $J \in \mathfrak{z}(\mathfrak{h})$, so $J_{0}=\left.\operatorname{ad}(J)\right|_{\mathfrak{m}}$. Since $J_{0}^{2}=-\mathrm{Id}$, we decompose $\mathfrak{m}^{\mathbb{C}}$ into $\pm i$-eigenspaces for $J_{0}: \mathfrak{m}^{\mathbb{C}}=\mathfrak{m}^{+}+\mathfrak{m}^{-}$. Both $\mathfrak{m}^{+}$and $\mathfrak{m}^{-}$are abelian, $\left[\mathfrak{h}^{\mathbb{C}}, \mathfrak{m}^{ \pm}\right] \subset \mathfrak{m}^{ \pm}$, and there are $\operatorname{Ad}(H)$-equivariant isomorphisms $\mathfrak{m} \cong \mathfrak{m}^{ \pm}$given by $X \mapsto \frac{1}{2}\left(X \mp i J_{0} X\right)$. 
Consider a maximal abelian subalgebra $\mathfrak{t}$ of $\mathfrak{h}$. Its complexification $\mathfrak{t}^{\mathbb{C}}$ gives a Cartan subalgebra of $\mathfrak{g}^{\mathbb{C}}$, for which we consider the root system $\Delta=\Delta\left(\mathfrak{g}^{\mathbb{C}}, \mathfrak{t}^{\mathbb{C}}\right)$ and the decomposition $\mathfrak{g}^{\mathbb{C}}=\mathfrak{t}^{\mathbb{C}}+\sum_{\alpha \in \Delta} \mathfrak{g}_{\alpha}^{\mathbb{C}}$. Since $\operatorname{ad}\left(\mathfrak{t}^{\mathbb{C}}\right)$ preserves $\mathfrak{h}^{\mathbb{C}}$ and $\mathfrak{m}^{\mathbb{C}}, \mathfrak{g}_{\alpha}^{\mathbb{C}}$ must lie either in $\mathfrak{h}^{\mathbb{C}}$ or in $\mathfrak{m}^{\mathbb{C}}$. If $\mathfrak{g}_{\alpha}^{\mathbb{C}} \subset \mathfrak{h}^{\mathbb{C}}$ (resp. $\mathfrak{g}_{\alpha}^{\mathbb{C}} \subset \mathfrak{m}^{\mathbb{C}}$ ) we say that the root $\alpha$ is compact (resp. non-compact). We choose an ordering of the roots in such a way that $\mathfrak{m}^{+}$(resp. $\mathfrak{m}^{-}$) is spanned by the root vectors corresponding to the non-compact positive (resp. negative) roots.

We denote by $\langle\cdot, \cdot\rangle$ the Killing form on $\mathfrak{g}^{\mathbb{C}}$. For each root $\alpha \in \Delta$, let $H_{\alpha} \in i \mathfrak{t}$ be the dual of $\alpha$, i.e.,

$$
\alpha(Y)=\left\langle Y, H_{\alpha}\right\rangle \quad \text { for } Y \in i \text { t. }
$$

Define, as usual, $h_{\alpha}=\frac{2 H_{\alpha}}{\left\langle H_{\alpha}, H_{\alpha}\right\rangle} \in i \mathfrak{t}$, and $e_{\alpha} \in \mathfrak{g}_{\alpha}^{\mathbb{C}}$ such that $\left[e_{\alpha}, e_{-\alpha}\right]=h_{\alpha}$. Two roots $\alpha, \beta \in \Delta$ are said to be strongly orthogonal if neither $\alpha+\beta$ nor $\alpha-\beta$ is a root (equivalently $\left[\mathfrak{g}^{\alpha}, \mathfrak{g}^{ \pm \beta}\right]=\{0\}$ ). A system of strongly orthogonal roots is a maximal set of strongly orthogonal positive non-compact roots. It has a number of elements equal to the rank $r=\operatorname{rk}(G / H)$ of the symmetric space $G / H$, i.e., the maximal dimension of a flat, totally geodesic submanifold of $G / H$. Moreover, for two strongly orthogonal roots $\gamma \neq \gamma^{\prime}$ we have

$$
\left[e_{ \pm \gamma}, e_{ \pm \gamma^{\prime}}\right]=0, \quad\left[e_{ \pm \gamma}, h_{\gamma^{\prime}}\right]=0 .
$$

For a strongly orthogonal system of roots $\Gamma$, consider

$$
x_{\Gamma}=\sum_{\gamma \in \Gamma} x_{\gamma}, \quad y_{\Gamma}=\sum_{\gamma \in \Gamma} y_{\gamma}, \quad e_{\Gamma}=\sum_{\gamma \in \Gamma} e_{\gamma}, \quad c=\exp \left(\frac{\pi}{4} i y_{\Gamma}\right) \in U \subset G^{\mathbb{C}},
$$

where $G^{\mathbb{C}}$ is the simply connected Lie group with Lie algebra $\mathfrak{g}^{\mathbb{C}}$ and $U$ is its compact real form (with Lie algebra $\mathfrak{h} \oplus i \mathfrak{m}$ ). We define the Cayley transform as the action of the element $c$ on the Lie algebra $\mathfrak{g}^{\mathbb{C}}$ by $\operatorname{Ad}(c): \mathfrak{g}^{\mathbb{C}} \rightarrow \mathfrak{g}^{\mathbb{C}}$. The Cayley transform $\operatorname{Ad}(c)$ satisfies $\operatorname{Ad}\left(c^{8}\right)=\operatorname{Id}, \operatorname{Ad}(c) \circ \theta=\theta \circ \operatorname{Ad}\left(c^{-1}\right)$ for the Cartan involution $\theta$, and consequently $\operatorname{Ad}\left(c^{4}\right)$ preserves $\mathfrak{h}$ and $\mathfrak{m}$, even though $\operatorname{Ad}(c)$ does not preserve $\mathfrak{g}$.

It is well-known that a Hermitian symmetric space of non-compact type $G / H$ can be realized as a bounded symmetric domain. For the classical groups this is due to Cartan, while the general case is given by the Harish-Chandra embedding $G / H \rightarrow \mathfrak{m}_{+}$ which defines a biholomorphism between $G / H$ and the bounded symmetric domain $\mathcal{D}$ given by the image of $G / H$ in the complex vector space $\mathfrak{m}_{+}$. Now, for any bounded domain $\mathcal{D}$ there is the Shilov boundary of $\mathcal{D}$ which is defined as the smallest closed subset $\check{S}$ of the topological boundary $\partial \mathcal{D}$ for which every function $f$ continuous on $\overline{\mathcal{D}}$ and holomorphic on $\mathcal{D}$ satisfies that

$$
|f(z)| \leq \max _{w \in \tilde{S}}|f(w)| \text { for every } z \in \mathcal{D} .
$$

The Shilov boundary $\check{S}$ is the unique closed $G$-orbit in $\partial \mathcal{D}$.

The simplest situation to consider is that of the hyperbolic plane. The Poincaré disc is its realization as a bounded symmetric domain. However, we know that the hyperbolic plane can also be realized as the Poincaré upper-half plane. There are other Hermitian symmetric spaces that, like the hyperbolic plane, admit a realization similar to the upper-half plane. These are the tube type symmetric spaces. 
Let $V$ be a real vector space and let $\Omega \subset V$ be an open cone in $V$. A tube over the cone $\Omega$ is a domain of the form

$$
T_{\Omega}=\left\{u+i v \in V^{\mathbb{C}}, u \in V, v \in \Omega\right\} .
$$

A domain $\mathcal{D}$ is said to be of tube type if it is biholomorphic to a tube $T_{\Omega}$. In the case of a symmetric domain the cone $\Omega$ is also symmetric. An important characterization of the tube type symmetric domains is given by the following.

Proposition 5.1. Let $\mathcal{D}$ be a bounded symmetric domain corresponding to the Hermitian symmetric space $G / H$. The following are equivalent:

(i) $\mathcal{D}$ is of tube type.

(ii) $\operatorname{dim}_{\mathbb{R}} \check{S}=\operatorname{dim}_{\mathbb{C}} \mathcal{D}$.

(iii) $\breve{S}$ is a symmetric space of compact type.

(iv) $\operatorname{Ad}\left(c^{4}\right)=\operatorname{Id}$.

There is a generalization of the Cayley map that sends the unit disc biholomorphically to the upper-half plane. Let $\mathcal{D}$ be the bounded domain associated to a Hermitian symmetric space $G / H$. Acting by a particular element in $G$, known as the Cayley element, one obtains a map

$$
c: \mathcal{D} \longrightarrow \mathfrak{m}^{+}
$$

which is called the Cayley transform. A relevant fact for us is the following.

Proposition 5.2. Let $\mathcal{D}$ be the symmetric domain corresponding to the Hermitian symmetric space $G / H$. Let $\mathcal{D}$ be of tube type. Then the image by the Cayley transform $c(\mathcal{D})$ is biholomorphic to a tube domain $T_{\Omega}$ where the symmetric cone $\Omega$ is the noncompact dual of the Shilov boundary of $\mathcal{D}$. In fact the Shilov boundary is a symmetric space isomorphic to $H / H^{\prime}$ for a certain subgroup $H^{\prime} \subset H$, and $\Omega=H^{*} / H^{\prime}$ is its non-compact dual symmetric space.

Proposition 5.3. (1) The symmetric spaces defined by $\operatorname{Sp}(2 n, \mathbb{R}), \mathrm{SO}_{0}(2, n)$ are of tube type.

(2) The symmetric space defined by $\mathrm{SU}(p, q)$ is of tube type if and only if $p=q$.

(3) The symmetric space defined by $\mathrm{SO}^{*}(2 n)$ is of tube type if and only if $n$ is even.

For a tube type classical irreducible symmetric space $G / H$, Table 5 indicates the Shilov boundary $\breve{S}=H / H^{\prime}$, its non-compact dual $\Omega=H^{*} / H^{\prime}$, the isotropy representation space $\mathfrak{m}^{\prime}$ and its complexification $\mathfrak{m}^{\mathbb{C}}$, corresponding to the Cartan decomposition of the Lie algebra $\operatorname{Lie}\left(H^{*}\right)=\mathfrak{h}^{\prime}+\mathfrak{m}^{\prime}$ of $H^{*}$. The vector space $\mathfrak{m}^{\prime}$ has the structure of a Euclidean Jordan algebra, where the cone $\Omega$ is realized.

The following lemma will be important for our Cayley correspondence.

Lemma 5.4. There are $\operatorname{Ad}\left(H^{\prime \mathbb{C}}\right)$-equivariant isomorphisms $\operatorname{ad}\left(e_{\Gamma}\right): \mathfrak{m}_{T}^{-} \rightarrow \mathfrak{m}^{\prime \mathbb{C}}$ and $\operatorname{ad}\left(e_{\Gamma}\right): \mathfrak{m}^{\prime \mathbb{C}} \rightarrow \mathfrak{m}_{T}^{+}$.

The study of certain problems in non-tube type domains can be reduced to the tube type thanks to the following. 
Proposition 5.5. Let $G / H$ be a Hermitian symmetric space of non-compact type. There exists a subgroup $\widetilde{G} \subset G$ such that $\widetilde{G} / \widetilde{H} \subset G / H$ is a maximal isometrically embedded symmetric space of tube type, where $\widetilde{H} \subset \widetilde{G}$ is a maximal compact subgroup.

Table ?? gives the maximal symmetric space of tube type isometrically embedded in the two series of irreducible classical symmetric spaces of non-tube type. We describe also the Shilov boundaries of $G / H$ and $\widetilde{G} / \widetilde{H}$ which are of the form $\breve{S}=H / H^{\prime}$, and $\widetilde{S}=\widetilde{H} / \widetilde{H}^{\prime}$, respectively. Notice that in the non-tube case the Shilov boundary $\check{S}$ is a homogeneous space $H / H^{\prime}$ but it is not symmetric.

5.2. The Toledo character. We introduce the Toledo character associated to a simple Lie algebra $\mathfrak{g}$ of Hermitian type as the character on the Lie algebra $\mathfrak{h}^{\mathbb{C}}$ given as follows.

Definition 5.6. The Toledo character $\chi_{T}: \mathfrak{h}^{\mathbb{C}} \rightarrow \mathbb{C}$ is defined, for $Y \in \mathfrak{h}^{\mathbb{C}}$ in terms of the Killing form, by

$$
\chi_{T}(Y)=\frac{1}{N}\langle-i J, Y\rangle
$$

where $N$ is the dual Coxeter number.

Since $J$ is in the center, $\chi_{T}$ vanishes on $\left[\mathfrak{h}^{\mathbb{C}}, \mathfrak{h}^{\mathbb{C}}\right]$, hence determines a character.

We study now when the Toledo character lifts to a character of the group $H^{\mathbb{C}}$. Note that this depends on the choice of the pair $(G, H)$ defining the same symmetric space. Let $Z_{0}^{\mathbb{C}}$ denote the identity component of $Z\left(H^{\mathbb{C}}\right)$.

Proposition 5.7. Define oJ to be the order of $e^{2 \pi J}$ and $\ell=\left|Z_{0}^{\mathbb{C}} \cap\left[H^{\mathbb{C}}, H^{\mathbb{C}}\right]\right|$. For $q \in \mathbb{Q}$, the character $q \chi_{T}$ lifts to $H^{\mathbb{C}}$ if and only if $q$ is an integral multiple of

$$
q_{T}=\frac{\ell N}{o_{J} \operatorname{dim} \mathfrak{m}} .
$$

The value of $q_{T}$ in the standard examples is given in Table 3. Note in particular that $q_{T}=\frac{1}{2}$ for all classical groups except $\mathrm{SO}^{*}$, for which $q_{T}=1$. So for all classical groups the Toledo character lifts to $H^{\mathbb{C}}$. For the adjoint group, $o_{J}=1$ so $q_{T}=\frac{\ell N}{\operatorname{dim} \mathfrak{m}}$. In the tube case $N=\frac{\operatorname{dimm}}{r}$ so this gives $q_{T}=\frac{\ell}{r}$. The values of $q_{T}$ in the non-tube case are given in Table 4.

Finally, the following lemma will prove later that the Toledo invariants defined from the two points of view of Higgs bundles and representations coincide.

Lemma 5.8. The Toledo character $\chi_{T}$ defines a symmetric Kähler form on $G / H$ by

$$
\omega(Y, Z)=i \chi_{T}([Y, Z]), \text { for } Y, Z \in \mathfrak{m},
$$

with minimal holomorphic sectional curvature -1 .

We define now for $G$ of tube type a determinant polynomial, det, on $\mathfrak{m}^{+}$, whose degree equals the rank of the symmetric space. This determinant is a familiar object in Jordan algebra theory [FK94], but it can be introduced in an elementary way as follows 
[KV79, Lemma 2.3]: it is the unique $H_{0}^{\prime}$-invariant polynomial on $\mathfrak{m}^{+}$which restricts on $\mathfrak{a}^{+}=\oplus_{1}^{r} \mathbb{C} e_{\gamma_{i}}$ to

$$
\operatorname{det} \sum_{1}^{r} \lambda_{i} e_{\gamma_{i}}=\prod_{1}^{r} \lambda_{i} .
$$

Here $H_{0}^{\prime}$ is the identity component of $H^{\prime}$. The existence comes from the Chevalley theorem on invariant polynomials, since the Weyl group acts exactly by all permutations on the $\left(e_{\gamma_{i}}\right)$ (see again $\left.[\mathrm{KV} 79]\right)$.

The main useful property for us is the following equivariance:

Lemma 5.9. Let $G$ be of tube type. For $h \in H^{\mathbb{C}}$ and $x \in \mathfrak{m}^{+}$we have

$$
\operatorname{det}(\operatorname{Ad}(h) x)=\tilde{\chi}_{T}(h) \operatorname{det}(x),
$$

where $\tilde{\chi}_{T}$ is the lifting of $\chi_{T}$ to $H^{\mathbb{C}}$.

Note that we implicitly assumed here that the lifting $\tilde{\chi}_{T}$ exists, otherwise the same identity remains true after taking power $q_{T}$.

We define a notion of rank for an element in $\mathfrak{m}^{+}$for $G$ of Hermitian type (tube or non-tube). Choose $\mathfrak{a}^{+}=\oplus_{1}^{r} \mathbb{C} e_{\gamma_{i}}$. Any element of $\mathfrak{m}^{+}$is conjugate under $H^{\mathbb{C}}$ to an element of $\mathfrak{a}^{+}$.

Definition 5.10. Let $x \in \mathfrak{m}^{+}$, and $y=\sum \lambda_{i} e_{\gamma_{i}} \in \mathfrak{a}^{+}$be conjugate to $x$ under $H^{\mathbb{C}}$. Then we say that $x$ has rank $r^{\prime}$ if $y$ has exactly $r^{\prime}$ non-zero coefficients.

This is well defined because the Weyl group acts only by permutations on $\mathfrak{a}^{+}$. Also, in the tube case, one can give a more intrinsic interpretation using the determinant: polarize the determinant to get an $r$-linear map $C$ on $\mathfrak{m}^{+}$such that $C(x, \ldots, x)=\operatorname{det}(x)$; then the rank of $x$ is the maximal integer $r^{\prime}$ such that the $\left(r-r^{\prime}\right)$-form $C(x, \ldots, x, \cdot, \ldots, \cdot)$ is not identically zero, which is clearly an invariant notion.

Remark 5.11. In the case of $\mathrm{SU}(p, q)$ the rank on $\mathfrak{m}^{+}$specializes to the notion of rank for a rectangular matrix $q \times p$. For $\operatorname{Sp}(2 n, \mathbb{R})$, the rank on $\mathfrak{m}^{+}$is the rank for an element of $S^{2}\left(\mathbb{C}^{n}\right)$ seen as an endomorphism.

The following proposition plays an important role in what follows.

Proposition 5.12. Let $1 \leq r^{\prime} \leq r$. The group $H^{\mathbb{C}}$ acts transitively on the set of elements of rank $r^{\prime}$ in $\mathfrak{m}^{+}$. In particular, the set of regular (that is maximal rank) elements in $\mathfrak{m}^{+}$is $H^{\mathbb{C}} / H^{\prime \mathbb{C}}$.

5.3. Toledo invariant and Milnor-Wood inequality. Let $X$ be a compact Riemann surface and let $(E, \varphi)$ be a $G$-Higgs bundle over $X$. The decomposition $\mathfrak{m}^{\mathbb{C}}=\mathfrak{m}^{+}+\mathfrak{m}^{-}$ gives a vector bundle decomposition $E\left(\mathfrak{m}^{\mathbb{C}}\right)=E\left(\mathfrak{m}^{+}\right) \oplus E\left(\mathfrak{m}^{-}\right)$and hence the Higgs field has two components:

$$
\varphi=\left(\varphi^{+}, \varphi^{-}\right) \in H^{0}\left(X, E\left(\mathfrak{m}^{+}\right) \otimes K\right) \oplus H^{0}\left(X, E\left(\mathfrak{m}^{-}\right) \otimes K\right)=H^{0}\left(X, E\left(\mathfrak{m}^{\mathbb{C}}\right) \otimes K\right) .
$$

When the group $G$ is a classical group, or more generally when $H$ is a classical group, it is useful to take the standard representation of $H^{\mathbb{C}}$ to describe a $G$-Higgs bundle in terms of associated vector bundles. This is the approach taken in [BGG03, BGG06, BGG15, GGM13]. 
Let $(E, \varphi)$ be a $G$-Higgs bundle. Consider the Toledo character $\chi_{T}$. Up to an integer multiple, $\chi_{T}$ lifts to a character $\tilde{\chi}_{T}$ of $H^{\mathbb{C}}$. Let $E\left(\tilde{\chi}_{T}\right)$ be the line bundle associated to $E$ via the character $\tilde{\chi}_{T}$.

Definition 5.13. We define the Toledo invariant $\tau$ of $(E, \varphi)$ by

$$
\tau=\tau(E):=\operatorname{deg}\left(E\left(\tilde{\chi}_{T}\right)\right) .
$$

If $\tilde{\chi}_{T}$ is not defined, but only $\tilde{\chi}_{T}^{q}$, one must replace the definition by $\frac{1}{q} \operatorname{deg} E\left(\tilde{\chi}_{T}^{q}\right)$.

We denote by $\mathcal{M}_{\tau}^{\alpha}(G)$ the subspace of $\mathcal{M}^{\alpha}(G)$ corresponding to $G$-Higgs bundles whose Toledo invariant equals $\tau$. For $\alpha=0$ we simplify our notation setting $\mathcal{M}_{\tau}(G):=$ $\mathcal{M}_{\tau}^{0}(G)$

The following proposition relates our Toledo invariant to the usual Toledo invariant of a representation, first defined in [Tol89].

Proposition 5.14. Let $\rho: \pi_{1}(X) \rightarrow G$ be reductive and let $(E, \varphi)$ be the corresponding polystable $G$-Higgs bundle given by Theorem 2.12. Let $f: \tilde{X} \rightarrow G / H$ be the corresponding harmonic metric. Then

$$
\tau(E)=\frac{1}{2 \pi} \int_{X} f^{*} \omega
$$

where $\omega$ is the Kähler form of the symmetric metric on $G / H$ with minimal holomorphic sectional curvature -1, computed in Lemma 5.8.

In particular, $\tau(E)$ is the Toledo invariant of $\rho$.

The Toledo invariant is related to the topological class of the bundle $E$ defined as an element of $\pi_{1}(H)$. To explain this, assume that $H^{\mathbb{C}}$ is connected. The topological classification of $H^{\mathbb{C}}$-bundles $E$ on $X$ is given by a characteristic class $c(E) \in \pi_{1}\left(H^{\mathbb{C}}\right)$ as follows. From the exact sequence

$$
1 \rightarrow \pi_{1}\left(H^{\mathbb{C}}\right) \rightarrow \widetilde{H^{\mathbb{C}}} \rightarrow H^{\mathbb{C}} \rightarrow 1
$$

we obtain a long exact sequence in cohomology and, in particular, the connection map

$$
H^{1}\left(X, \underline{H}^{\mathbb{C}}\right) \stackrel{c}{\rightarrow} H^{2}\left(X, \pi_{1}\left(H^{\mathbb{C}}\right)\right),
$$

where $\underline{H}^{\mathbb{C}}$ is the sheaf of local holomorphic functions in $X$ with values in $H^{\mathbb{C}}$. The cohomology set $H^{1}\left(X, \underline{H}^{\mathbb{C}}\right)$ (not necessarily a group since $H^{\mathbb{C}}$ is in general not abelian) parametrizes isomorphism classes of principal $H^{\mathbb{C}}$-bundles over $X$. On the other hand, since $\operatorname{dim}_{\mathbb{R}} X=2$, by the universal coefficient theorem and the fact that the fundamental group of a Lie group is abelian, $H^{2}\left(X, \pi_{1}\left(H^{\mathbb{C}}\right)\right)$ is isomorphic to $\pi_{1}\left(H^{\mathbb{C}}\right)$. Moreover, $\pi_{1}\left(H^{\mathbb{C}}\right) \cong \pi_{1}(H) \cong \pi_{1}(G)$ since $H$ is a deformation retract for both $H^{\mathbb{C}}$ and $G$. This map thus associates a topological invariant in $\pi_{1}(H)$ to any $G$-Higgs bundle on $X$.

By the relation between the fundamental group and the centre of a Lie group, the topological class in $\pi_{1}(H)$ is of special interest when $H$ has a non-discrete centre, i.e., when $G$ is of Hermitian type. In this case, $\pi_{1}(H)$ is isomorphic to $\mathbb{Z}$ plus possibly a torsion group (among the classical groups, $\mathrm{SO}_{0}(2, n)$ is the only one with torsion). Very often (see for example [BGG06]), the Toledo invariant of a $G$-Higgs bundle $(E, \varphi)$ is defined as the projection of $c(E)$ defined by $(5.4)$ on the torsion-free part, $\mathbb{Z}$. The general relation is the following. 
Proposition 5.15. Let $(E, \varphi)$ be a $G$-Higgs bundle, and $d \in \mathbb{Z}$ the projection on the torsion-free part of the class $c(E)$ defined by (5.4). Then $d$ is related to the Toledo invariant by

$$
\tau=\frac{d}{q_{T}} .
$$

Definition 5.10 gives the ranks of $\varphi^{+}$and $\varphi^{-}$at a point $x \in X$. The space of elements of $\mathfrak{m}^{ \pm}$with rank at most $\rho$ is an algebraic subvariety of $\mathfrak{m}^{ \pm}$, so the ranks of $\varphi^{+}$and $\varphi^{-}$ are the same at all points of $X$ except a finite number of points where it it smaller. We therefore have a well defined notion of rank of $\varphi^{+}$and $\varphi^{-}$:

Definition 5.16. The generic value on $X$ of the rank of $\varphi^{+}$is called the rank of $\varphi^{+}$ and denoted $\operatorname{rk} \varphi^{+}$. Analogously we define the rank $\operatorname{rk} \varphi^{-}$of $\varphi^{-}$.

The main result of this section is the following.

Theorem 5.17. Let $\left(E, \varphi^{+}, \varphi^{-}\right)$be a semistable G-Higgs bundle. Then, the Toledo invariant of $E$ satisfies:

$$
-\operatorname{rk}\left(\varphi^{+}\right)(2 g-2) \leq \tau \leq \operatorname{rk}\left(\varphi^{-}\right)(2 g-2) .
$$

In particular, we obtain the familiar Milnor-Wood inequality

$$
|\tau| \leq \operatorname{rk}(G / H)(2 g-2),
$$

and equality holds if and only if $\varphi^{+}\left(\right.$resp. $\left.\varphi^{-}\right)$is regular at each point in the case $\tau<0$ (resp. $\tau>0$ ).

Theorem 5.17 was proved on a case by case basis for the classical groups [?, Got01, BGG03, BGG06, BGG15, GGM13]. In these references, the bound given is for the integer $d \in \pi_{1}(H) \cong \pi_{1}\left(H^{\mathbb{C}}\right) \cong \mathbb{Z}$ associated naturally to the $H^{\mathbb{C}}$-bundle $E$. This differs from the Toledo invariant by a rational multiple. From Table 3 and Proposition 5.15 combined with Theorem 5.17 we obtain the Milnor-Wood inequalities given in [BGG06] for the classical Hermitian groups. Our intrinsic general approach covers of course the exceptional groups and quotients and covers of classical groups that have not been studied previously.

A polystable $G$-Higgs bundle $(E, \varphi)$ is, by Theorem 2.12 , in correspondence with a reductive representation $\rho: \pi_{1}(X) \rightarrow G$, and from Proposition 5.14 the Toledo invariant of $(E, \varphi)$ coincides with the Toledo invariant of a representation of the fundamental group in $G$. In the context of representations the inequality $|\tau| \leq \operatorname{rk}(G / H)(2 g-2)$, goes back to Milnor [Mil58], who studies the case $G=\operatorname{PSL}(2, \mathbb{R})$, and was proved in various cases in [Woo71, Dup78, DT87, CO03], and in general in [BIW10]. We should point out that the Higgs bundle approach gives the Milnor-Wood inequality for an arbitrary representation, as the other approaches do, since such a representation can always be deformed to a reductive one.

5.4. Hermitian groups of tube type and Cayley correspondence. We define a polystable Higgs bundle $(E, \varphi)$ to be maximal if its Toledo invariant $\tau$ attains one of the bounds of the inequality i.e., $\tau= \pm r(2 g-2)$, where $r=\operatorname{rk}(G / H)$. We denote $\tau_{\max }=\operatorname{rk}(G / H)(2 g-2)$. 
Let $H^{*}$ be the non-compact dual of $H$ as defined in Proposition 5.2. In this section we establish a bijective correspondence between maximal $G$-Higgs bundles over $X$ and $K^{2}$-twisted $H^{*}$-Higgs bundles over $X$, as defined in Remark 2.5, where $K^{2}$ is the square of the canonical line bundle.

Suppose that $(E, \varphi)$ is a polystable maximal $G$-Higgs bundle, and choose for example $\tau=-r(2 g-2)$. By Theorem 5.17, the field $\varphi^{+}$has rank $r$ at each point. Let $Z_{0}^{\mathbb{C}} \simeq \mathbb{C}^{*}$ be the connected component of the identity of the center of $H^{\mathbb{C}}$. There is an exact sequence

$$
1 \longrightarrow Z_{0}^{\mathbb{C}} \longrightarrow H^{\mathbb{C}} \longrightarrow H^{\mathbb{C}} / Z_{0}^{\mathbb{C}} \longrightarrow 1
$$

so there is an action of $Z_{0}^{\mathbb{C}}$-bundles on $H^{\mathbb{C}}$-bundles that we will denote by $\otimes$ : in this way, if $\kappa$ is a line bundle over $X$, we can define $E \otimes \kappa$ (here we are identifying the line bundle $\kappa$ with its corresponding $\mathbb{C}^{*}$-bundle).

If $\kappa$ is an $o_{J}$-root of $K$, where $o_{J}$ is the order of $e^{2 \pi J}$, then $\varphi^{+}$defines a reduction of the $H^{\mathbb{C}}$-bundle $E \otimes \kappa$ to the group $H^{\prime \mathbb{C}}$.

Of course, such $\kappa$ exists only if $o_{J}$ divides $2 g-2$. We will now suppose that $\kappa$ exists and is fixed. Denote by $E^{\prime}$ the reduction of $E \otimes \kappa$ to $H^{\prime \mathbb{C}}$. As we have seen, $\varphi^{+} \in H^{0}\left(X, E^{\prime}\left(\mathfrak{m}^{+}\right)\right)$, and similarly $\varphi^{-} \in H^{0}\left(X, E^{\prime}\left(\mathfrak{m}^{-}\right) \otimes K^{2}\right)$. From Lemma 5.4, we have an isomorphism

$$
\operatorname{ad} \varphi^{+}: E^{\prime}\left(\mathfrak{m}^{-}\right) \longrightarrow E^{\prime}\left(\mathfrak{m}^{\prime \mathbb{C}}\right)
$$

so that we can define a Higgs field

$$
\varphi^{\prime}=\left[\varphi^{+}, \varphi^{-}\right] \in H^{0}\left(X, E^{\prime}\left(\mathfrak{m}^{\prime \mathbb{C}}\right) \otimes K^{2}\right) .
$$

The data $\left(E^{\prime}, \varphi^{\prime}\right)$ is a $K^{2}$-twisted $H^{*}$-Higgs bundle.

Conversely, from a $K^{2}$-twisted $H^{*}$-Higgs bundle $\left(E^{\prime}, \varphi^{\prime}\right)$ we can reconstruct $(E, \varphi)$ in the following way. The bundle is $E=E^{\prime} \otimes \kappa^{-1}$. Observe that for the $H^{\prime \mathbb{C}_{\text {-bundle }} E^{\prime}}$ we have canonical section $e_{\Gamma} \in H^{0}\left(X, E^{\prime}\left(\mathfrak{m}^{+}\right)\right)$corresponding to the element $e_{\Gamma} \in \mathfrak{m}^{+}$ fixed by $H^{\prime}$, which becomes by Lemma 5.4 a section $\varphi^{+} \in H^{0}\left(X, E\left(\mathfrak{m}^{+}\right) \otimes K\right)$. Finally, $\varphi^{-}$is reconstructed from $(5.6)$ as $\left(\operatorname{ad} \varphi^{+}\right)^{-1}\left(\varphi^{\prime}\right)$. Therefore, $\kappa$ being fixed, we obtain a complete correspondence between maximal $G$-Higgs bundles and $K^{2}$-twisted $H^{*}$-Higgs bundles. We refer to $\left(E^{\prime}, \varphi^{\prime}\right)$ as the Cayley partner of $(E, \varphi)$. One hs the following.

Theorem 5.18 (Cayley correspondence). Let $G$ be a connected non-compact real simple Hermitian Lie group of tube type with finite centre. Let $H$ be a maximal compact subgroup of $G$ and $H^{*}$ be the non-compact dual of $H$ in $H^{\mathbb{C}}$. Let $J$ be the element in $\mathfrak{z}$ (the centre of $\mathfrak{h}$ ) defining the almost complex structure on $\mathfrak{m}$. If the order of $e^{2 \pi J} \in H^{\mathbb{C}}$ divides $(2 g-2)$, then there is an isomorphism of complex algebraic varieties

$$
\mathcal{M}_{\max }(G) \cong \mathcal{M}_{K^{2}}\left(H^{*}\right)
$$

given by $(E, \varphi) \mapsto\left(E^{\prime}, \varphi^{\prime}\right)$ as above.

Remark 5.19. The condition $o_{J} \mid(2 g-2)$ is always satisfied for a group of adjoint type, since in this case $o_{J}=1$. Table 3 shows that the $o_{J}$ divides $(2 g-2)$ for the classical and exceptional groups. This may not happen for coverings of these groups, where $o_{J}$ may be bigger. 


\section{6. $\mathrm{SO}(p, q)$-Higgs BUNDles AND Higher TeICHMÜlleR SPACES}

Details on this section can be found in $[\mathrm{ABC}+18, \mathrm{ABC}+18 \mathrm{~b}]$.

6.1. $\mathrm{SO}(p, q)$-Higgs bundles and topological invariants. An $\mathrm{SO}(p, q)$-Higgs bundle on $X$ is equivalent to a triple $(V, W, \eta)$ where $V$ and $W$ are respectively rank $p$ and rank $q$ vector bundles with orthogonal structures such that $\operatorname{det}(W) \simeq \operatorname{det}(V)$, and $\eta$ is a holomorphic bundle map $\eta: W \rightarrow V \otimes K$.

The cases for $p \leq 2$ are somewhat special. For $p>2$, rank $p$ orthogonal bundles on $X$ are classified topologically by their first and second Stiefel-Whitney classes, $s w_{1} \in$ $H^{1}\left(X, \mathbb{Z}_{2}\right)$ and $s w_{2} \in H^{2}\left(X, \mathbb{Z}_{2}\right)$. These primary topological invariants are constant on connected components of the moduli space $\mathcal{M}(\mathrm{SO}(p, q))$. Since $\operatorname{det}(W) \simeq \operatorname{det}(V)$, it follows that $s w_{1}(V)=s w_{1}(W)$. The components of the moduli space $\mathcal{M}(\operatorname{SO}(p, q))$ are thus partially labeled by triples $(a, b, c) \in \mathbb{Z}_{2}^{2 g} \times \mathbb{Z}_{2} \times \mathbb{Z}_{2}$, where

$$
a=s w_{1}(V) \in H^{1}\left(X, \mathbb{Z}_{2}\right), \quad b=s w_{2}(V) \in H^{2}\left(X, \mathbb{Z}_{2}\right) \quad \text { and } \quad c=s w_{2}(W) \in H^{2}\left(X, \mathbb{Z}_{2}\right) .
$$

Using the notation $\mathcal{M}^{a, b, c}(\mathrm{SO}(p, q))$ to denote the union of components labeled by $(a, b, c)$, we can thus write

$$
\mathcal{M}(\mathrm{SO}(p, q))=\coprod_{(a, b, c) \in \mathbb{Z}_{2}^{2 g} \times \mathbb{Z}_{2} \times \mathbb{Z}_{2}} \mathcal{M}^{a, b, c}(\mathrm{SO}(p, q)) .
$$

Stability for $\mathrm{SO}(p, q)$-Higgs bundles implies that a Higgs bundle $(V, W, \eta)$ with $\eta=0$ is polystable if and only if $V$ and $W$ are both polystable orthogonal bundles. This leads to the immediate identification of one connected component in each space $\mathcal{M}^{a, b, c}(\mathrm{SO}(p, q))$. We use the subscript 'top' to designate these components, which contain $\mathrm{SO}(p, q)$-Higgs bundles with vanishing Higgs field.

Proposition 6.1. Assume that $2<p \leq q$. For every $(a, b, c) \in \mathbb{Z}_{2}^{2 g} \times \mathbb{Z}_{2} \times \mathbb{Z}_{2}$ the space $\mathcal{M}^{a, b, c}(\mathrm{SO}(p, q))$ has a non-empty connected component, denoted by $\mathcal{M}_{\mathrm{top}}^{a, b, c}(\mathrm{SO}(p, q))$, in which every point can be continuously deformed to the isomorphism class of an $\mathrm{SO}(p, q)$ Higgs bundle of the form $(V, W, \eta=0)$ where $V$ and $W$ are polystable orthogonal bundles.

We define

$$
\mathcal{M}_{\mathrm{top}}(\mathrm{SO}(p, q))=\coprod_{a, b, c} \mathcal{M}_{\mathrm{top}}^{a, b, c}(\mathrm{SO}(p, q))
$$

Remark 6.2. In the case $p=2$ it is no longer true that $\mathcal{M}_{\text {top }}^{a, b, c}(\operatorname{SO}(p, q))$ is non-empty for all $(a, b, c)$. In particular, if $a=0$, then $V=L \oplus L^{-1}$ which (a) is polystable if $\operatorname{deg} L=0$ and (b) has $s w_{2}(V)=\operatorname{deg} L \bmod 2$. Thus $\left.\mathcal{M}_{\mathrm{top}}^{0, b, c} \mathrm{SO}(2, q)\right)$ is empty if $b \neq 0$.

The main result in $[\mathrm{ABC}+18, \mathrm{ABC}+18 \mathrm{~b}]$ is that the moduli space $\mathcal{M}(\mathrm{SO}(p, q))$ has additional 'exotic' components disjoint from the components of $\mathcal{M}_{\text {top }}(\mathrm{SO}(p, q))$. These exotic components are identified as products of moduli spaces of so-called $L$-twisted Higgs bundles, where in each factor $L$ is a positive power of the canonical bundle $K$.

Let $L$ be a fixed holomorphic line bundle on $X$. An $L$-twisted $\mathrm{SO}(1, n)$-Higgs bundle on $X$ is equivalent to a triple $\left(I, W_{0}, \eta\right)$, where $W_{0}$ is a $\mathrm{O}(n, \mathbb{C})$-bundle, $I$ is the rank one orthogonal bundle $\operatorname{det}\left(W_{0}\right)$ and $\eta: W_{0} \rightarrow I \otimes L$ is a holomorphic bundle map. 
We get a decomposition similar to (6.1), namely

$$
\mathcal{M}_{K^{p}}(\mathrm{SO}(1, n))=\coprod_{(a, c) \in \mathbb{Z}_{2}^{2 g} \times \mathbb{Z}_{2}} \mathcal{M}_{K^{p}}^{a, c}(\mathrm{SO}(1, n))
$$

where $\mathcal{M}_{K^{p}}^{a, c}(\mathrm{SO}(1, n))$ denotes the component in which the $\mathrm{SO}(1, n)$-Higgs bundles are of the form $\left(I, W_{0}, \eta\right)$, with $a=s w_{1}\left(W_{0}\right)$ and $c=s w_{2}\left(W_{0}\right)$.

6.2. The case $2<p \leq q$. We can now state the main result in $[\mathrm{ABC}+18, \mathrm{ABC}+18 \mathrm{~b}]$. Theorem 6.3. Fix integers $(p, q)$ such that $2<p<q-1$. For each choice of $a \in \mathbb{Z}_{2}^{2 g}$ and $c \in \mathbb{Z}_{2}$, the moduli space $\mathcal{M}(\mathrm{SO}(p, q))$ has a connected component disjoint from $\mathcal{M}_{\text {top }}(\mathrm{SO}(p, q))$. This component is isomorphic to

$$
\mathcal{M}_{K^{p}}^{a, c}(\mathrm{SO}(1, q-p+1)) \times \mathcal{M}_{K^{2}}\left(\mathrm{SO}_{0}(1,1)\right) \times \cdots \times \mathcal{M}_{K^{2 p-2}}\left(\mathrm{SO}_{0}(1,1)\right),
$$

and lies in the sector $\mathcal{M}^{\alpha, 0, c}(\mathrm{SO}(p, q))$ where $\alpha=a$ if $p$ is odd and $\alpha=0$ if $p$ is even. Moreover, $\mathcal{M}(\mathrm{SO}(p, q))$ has no other connected components.

Remark 6.4. The group $\mathrm{SO}_{0}(1,1)$ is the connected component of the identity in $\mathrm{SO}(1,1)$, and $\mathcal{M}_{K^{2 j}}\left(\mathrm{SO}_{0}(1,1)\right)$ can be identified with $H^{0}\left(K^{2 j}\right)$. Thus, we can replace (6.4) with

$$
\mathcal{M}_{K^{p}}^{a, c}(\mathrm{SO}(1, q-p+1)) \times \bigoplus_{j=1}^{p-1} H^{0}\left(K^{2 j}\right) .
$$

Remark 6.5. The existence of the exotic components described by (6.4) was proven for $p=2$ in [BGG06]. They are the exotic components with maximal Toledo invariant arising from Cayley correspondence (see Section 6.5). In particular, Theorem 6.3 can be viewed as a generalized Cayley correspondence. Contrary to the cases $p>2$, there are components of $\mathcal{M}(\mathrm{SO}(2, q))$ which are not in the family described by the theorem and also not in $\mathcal{M}_{\text {top }}(\mathrm{SO}(2, q))$. These are the components with non-maximal and non-zero Toledo invariant.

Corollary 6.6. For $2<p<q-1$, the moduli space $\mathcal{M}(\mathrm{SO}(p, q))$ has $3 \times 2^{2 g+1}$ connected components, $2^{2 g+1}$ of which are exotic components disjoint from $\mathcal{M}_{\text {top }}(\mathrm{SO}(p, q))$.

6.3. The case $q=p+1$. If $q=p+1$, then $\mathcal{M}_{K^{p}}^{a, c}(\operatorname{SO}(1, q-p+1))=\mathcal{M}_{K^{p}}^{a, c}(\operatorname{SO}(1,2))$, which is not always connected. Indeed, if $a=0$, then the Higgs bundles represented in $\mathcal{M}_{K^{p}}^{0, c}(\mathrm{SO}(1,2))$ can be taken to be of the form $\left(\mathcal{O}, L \oplus L^{-1}, \eta\right)$, where $L$ is a non-negative degree $d$ line bundle. Stability considerations impose a bound on $d$ so that

$$
\mathcal{M}_{K^{p}}^{0, c}(\mathrm{SO}(1,2))=\coprod_{\substack{0 \leq d \leq p(2 g-2) \\ d=c(\bmod 2)}} \mathcal{M}_{K^{p}}^{d}(\mathrm{SO}(1,2)) .
$$

Moreover, Collier [Col17] has showon that for each integer $d \in(0,2 g-2]$, the moduli space $\mathcal{M}_{K^{p}}^{d}(\mathrm{SO}(1,2))$ is diffeomorphic to a vector bundle of rank $d+g-1$ over the $(2 g-2-d)^{t h}$-symmetric product $\mathrm{Sym}^{2 g-2+d}(X)$. In particular, the components $\mathcal{M}_{K^{p}}^{d}(\mathrm{SO}(1,2))$ are smooth and connected.

The moduli spaces $\mathcal{M}(\mathrm{SO}(p, p+1))$ have been analyzed by Collier [Col17]. It was shown there that the topological invariants for $\mathrm{SO}(p, p+1)$-Higgs bundles, i.e. the triples $(a, b, c)$, do not distinguish all connected components. Two families of exotic components were identified. The components in the first family are labeled by an integer, $d$, in the 
range $0 \leq d \leq p(2 g-2)$, while those in the second family are labeled by a pair $(a, c) \in\left(\mathbb{Z}^{2 g}-\{0\}\right) \times \mathbb{Z}_{2}$. Though not described in this way in [Col17], these families can be identified as follows:

- In the the family labeled by $d$, each member is isomorphic to

$$
\mathcal{M}_{K^{p}}^{d}(\mathrm{SO}(1,2)) \times \mathcal{M}_{K^{2}}\left(\mathrm{SO}_{0}(1,1)\right) \times \cdots \times \mathcal{M}_{K^{2 p-2}}\left(\mathrm{SO}_{0}(1,1)\right),
$$

where $\mathcal{M}_{K^{p}}^{d}(\mathrm{SO}(1,2))$ is one of the components of $\mathcal{M}_{K^{p}}^{0, c}(\mathrm{SO}(1,2))$ as in (6.6).

- In the family labeled by $(a, c)$, each member is isomorphic to

$$
\mathcal{M}_{K^{p}}^{a, c}(\mathrm{SO}(1,2)) \times \mathcal{M}_{K^{2}}\left(\mathrm{SO}_{0}(1,1)\right) \times \cdots \times \mathcal{M}_{K^{2 p-2}}\left(\mathrm{SO}_{0}(1,1)\right) .
$$

The components are thus precisely those identified by Theorem 6.3 in the case $q=p+1$. The component count in this case is, however, different from the case $q>p+1$.

Corollary 6.7. For $p>2$, the moduli space $\mathcal{M}(\mathrm{SO}(p, p+1))$ has $3 \times 2^{2 g+1}+2 p(g-1)-1$ connected components. Among those, there are $2^{2 g+1}+2 p(g-1)-1$ 'exotic' components which are disjoint from $\mathcal{M}_{\text {top }}(\mathrm{SO}(p, p+1))$.

6.4. The case $q=p$. In this case $\mathcal{M}_{K^{p}}(\mathrm{SO}(1, q-p+1))=\mathcal{M}_{K^{p}}(\mathrm{SO}(1,1))$. A $K^{p}$ twisted $\mathrm{SO}(1,1)$-Higgs bundle consists of a triple $(I, I, \eta)$ where $I$ is a square root of the trivial bundle $\mathcal{O}$ and $\eta \in H^{0}\left(K^{p}\right)$. Such Higgs bundles are labeled by a single Stiefel-Whitney class, namely $a=s w_{1}(I)$, so that

$$
\mathcal{M}_{K^{p}}(\mathrm{SO}(1,1))=\coprod_{a \in H^{1}\left(X, \mathbb{Z}_{2}\right)} \mathcal{M}_{K^{p}}^{a}(\mathrm{SO}(1,1)) .
$$

With $q=p$, Theorem 6.3 thus gives $2^{2 g}$ exotic components of $\mathcal{M}(\mathrm{SO}(p, p))$ isomorphic to the moduli spaces

$$
\mathcal{M}_{K^{p}}^{a}(\mathrm{SO}(1,1)) \times \mathcal{M}_{K^{2}}\left(\mathrm{SO}_{0}(1,1)\right) \times \cdots \times \mathcal{M}_{K^{2 p-2}}\left(\mathrm{SO}_{0}(1,1)\right) .
$$

For each $a$, we can identify $\mathcal{M}_{K^{p}}^{a}(\mathrm{SO}(1,1))$ with $H^{0}\left(K^{p}\right)$. Thus, each exotic component is isomorphic to $H^{0}\left(K^{p}\right) \oplus \bigoplus_{j=1}^{p-1}\left(H^{0}\left(K^{2 j}\right)\right.$. This recovers the Hitchin component in $\mathcal{M}\left(\mathrm{SO}_{0}(p, p)\right)$ when $a=0$.

6.5. The case $p=2<q$. An $\mathrm{SO}(2, q)$-Higgs bundle is defined by a triple $(V, W, \eta)$ in which $V$ is an $\mathrm{O}(2, \mathbb{C})$-bundle. If $s w_{1}(V)=0$, i.e. if the structure group of $V$ reduces to $\mathrm{SO}(2, \mathbb{C})$, then $V$ can be assumed to be a direct sum of line bundles of the form $V=L \oplus L^{-1}$, with orthogonal structure given $q_{V}=\left(\begin{array}{ll}0 & 1 \\ 1 & 0\end{array}\right)$ in this splitting. Note that the second Stiefel-Whitney class of the orthogonal bundle $L \oplus L^{-1}$ is given by $s w_{2}=d(\bmod 2)$ where $d=\operatorname{deg}(L) \geq 0$.

For the groups $\mathrm{SO}(2, q)$, the connected components of the identity are isometry groups of Hermitian symmetric spaces of non-compact type. As explined in Section 5, the Higgs bundles have an associated Toledo invariant which, up to a normalization constant, is integer-valued but subject to a Milnor-Wood bound. For an $\mathrm{SO}_{0}(2, q)$-Higgs bundle 
( $\left.L \oplus L^{-1}, W, \eta\right)$, the Toledo invariant is basically the degree $d$ of $L$ and the MilnorWood bound is $0 \leq d \leq 2 g-2$. We thus get

$$
\mathcal{M}^{0, b, c}(\mathrm{SO}(2, q))=\coprod_{\substack{0 \leq d \leq 2 g-2 \\ d=b(\bmod 2)}} \mathcal{M}^{d, c}\left(\mathrm{SO}_{0}(2, q)\right)
$$

where $\mathcal{M}^{d, c}(\operatorname{SO}(2, q))$ denotes the component in which $\operatorname{deg}(L)=d$ and $s w_{2}(W)=c$.

The components where $d=2 g-2$ specializes further because in these components:

(1) $L$ has to be isomorphic to $K I$ where $I^{2}=\mathcal{O}$, and

(2) $W$ decomposes as $W=I \oplus W_{0}$ where $W_{0}$ is a rank $q-1$ orthogonal bundle with $s w_{1}\left(W_{0}\right)=I$.

As shown in [BGG06, CTT17], an $\mathrm{SO}(2, q)$-Higgs bundle with $L=K I, W=I \oplus W_{0}$ and $\eta=\left[q_{2}, \beta\right]: I \oplus W_{0} \rightarrow K I$ is defined by a $K^{2}$-twisted $\mathrm{SO}(1, q-1)$-Higgs bundle $\left(I, W_{0}, \beta\right)$ together with a quadratic differential $q_{2}$. Denoting $\mathcal{M}^{2 g-2, c}(\mathrm{SO}(2, q))$ by $\mathcal{M}_{\max }^{c}(\mathrm{SO}(2, q))$ it follows that

$\mathcal{M}_{\max }^{c}(\mathrm{SO}(2, q))=\coprod_{a \in H^{1}\left(X, \mathbb{Z}_{2}\right)} \mathcal{M}_{K^{2}}^{a, c}(\mathrm{SO}(1, q-1)) \times H^{0}\left(K^{2}\right)=\coprod_{a} \mathcal{M}_{K^{2}}^{a, c}\left(\mathrm{SO}(1, q-1) \times \mathrm{SO}_{0}(1,1)\right)$

where $a=s w_{1}(I)$. We thus get

$$
\mathcal{M}^{0,0, c}(\mathrm{SO}(2, q))=\coprod_{\substack{0 \leq d<2 g-2 \\ d=0(\bmod 2)}} \mathcal{M}^{d, c}(\mathrm{SO}(2, q)) \sqcup \coprod_{a} \mathcal{M}_{K^{2}}^{a, c}\left(\mathrm{SO}(1, q-1) \times \mathrm{SO}_{0}(1,1)\right)
$$

The group $\mathrm{SO}(1, q-1) \times \mathrm{SO}_{0}(1,1)$ is the Cayley partner to $\mathrm{SO}(2, q)$ and the objects in $\mathcal{M}_{K^{2}}^{a, c}\left(\mathrm{SO}(1, q-1) \times \mathrm{SO}_{0}(1,1)\right)$ are the Cayley partners to the Higgs bundles in $\mathcal{M}_{\max }^{c}(\mathrm{SO}(2, q))$. As shown in Section 5.4 Such Cayley partners are known to emerge in maximal components of $\mathcal{M}(\mathrm{G})$ whenever $\mathrm{G}$ is the isometry group of a Hermitian symmetric space of tube type. Comparing to Theorem 6.3, we see that the exotic components in $\mathcal{M}(\mathrm{SO}(p, q))$ are direct generalizations of these Cayley partners to the maximal components $\mathcal{M}_{\max }^{c}(\mathrm{SO}(2, q))$.

6.6. Cayley correspondence. Theorem 6.3 shows not only that additional exotic components exist, but also gives a model which describes them. Indeed, given the model, the result is proved directly by constructing a suitable map from the model to the moduli space $\mathcal{M}(\mathrm{SO}(p, q))$. The model is itself built from moduli spaces (of $K^{j}$ twisted Higgs bundles), so that in both the domain and target of our map the points represent equivalence classes of objects. one first describes a map between the objects, and then shows that it descends to the appropriate moduli spaces where it defines a homeomorphism onto a connected component.

The map relies in part on a parameterization of the Hitchin components of the moduli spaces $\mathcal{M}(\mathrm{SO}(p-1, p))$. Viewing theses moduli spaces as subspaces of $\mathcal{M}(\mathrm{SO}(2 p-1, \mathbb{C}))$, the parameterization is given by a section of the Hitchin fibration for $\mathcal{M}(\mathrm{SO}(2 p-$ $1, \mathbb{C})$ ). As explained in Section 4.3, the fibration is defined by $\mathrm{SO}(2 p-1, \mathbb{C})$-invariant polynomials evaluated on the Higgs field, giving a map to $\bigoplus_{j=1}^{p-1} H^{0}\left(K^{2 j}\right)$, and admits 
sections which parameterize connected components of $\mathcal{M}(\mathrm{SO}(p-1, p)) \subset \mathcal{M}(\mathrm{SO}(2 p-$ $1), \mathbb{C})$.

The $\mathrm{SO}(p, p-1)$-Higgs bundles in the image of the section can be taken to be of the form $\left(\mathcal{K}_{p}, \mathcal{K}_{p-1}, \sigma\left(q_{2}, q_{4}, \ldots, q_{2 p-2}\right)\right)$ where

$$
\mathcal{K}_{p}=K^{p-1} \oplus K^{p-3} \oplus \cdots \oplus K^{1-p}
$$

and $\sigma$ is given by a map

$$
\sigma: \bigoplus_{j=1}^{n} H^{0}\left(K^{2 j}\right) \rightarrow \operatorname{Hom}\left(\mathcal{K}_{p}, \mathcal{K}_{p-1}\right) \otimes K
$$

Theorem 6.3 is a consequence of the following (see $[\mathrm{ABC}+18, \mathrm{ABC}+18 \mathrm{~b}]$ ).

Theorem 6.8. Let $\left(I, W_{0}, \eta_{p}\right)$ be a $K^{p}$-twisted $\mathrm{SO}(1, q-p+1)$-Higgs bundle and take differentials $q_{2 j} \in H^{0}\left(K^{2 j}\right)$ for $j=1, \ldots p-1$. Using the notation from (6.14), consider the $\mathrm{SO}(p, q)$-Higgs bundle $(V, W, \eta)$ defined by

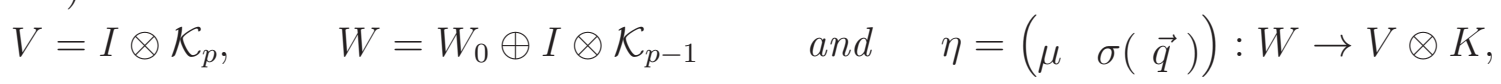

where $\mu=\left(\begin{array}{c}\eta_{p} \\ 0 \\ \vdots \\ 0\end{array}\right): W_{0} \rightarrow \mathcal{K}_{p} \otimes I \otimes K, \vec{q}=\left(q_{2}, \ldots, q_{2 p-2}\right)$ and $\sigma$ is the map from (6.15).

(1) $(V, W, \eta)$ is polystable if and only if $\left(I, W_{0}, \mu\right)$ is polystable. The map

$$
\left(\left(I, W_{0}, \mu\right) ; q_{2}, q_{4}, \ldots, q_{2 p-2}\right) \longmapsto(V, W, \eta)
$$

thus descends to define a map

$$
\Psi: \mathcal{M}_{K^{p}}(\mathrm{SO}(1, q-p+1)) \times \prod_{i=1}^{p-1} \mathcal{M}_{K^{2 i}}\left(\mathrm{SO}_{0}(1,1)\right) \longrightarrow \mathcal{M}(\mathrm{SO}(p, q)) .
$$

(2) The map $\Psi$ is injective.

(3) The image of $\Psi$ is open and closed.

(4) The image of $\Psi$ is disjoint from the components $\mathcal{M}_{0}(\mathrm{SO}(p, q))$.

There are several consequences of the results above for the moduli space of representations $\mathcal{R}(\mathrm{SO}(p, q))$. Recall that a representation $\rho: \pi_{1}(S) \rightarrow \mathrm{SO}_{0}(2,1)$ is called Fuchsian if it is discrete and faithful and that, since $\mathrm{SO}_{0}(p, p-1)$ is a split group of adjoint type, there is a unique principal embedding

$$
\iota: \mathrm{SO}_{0}(2,1) \rightarrow \mathrm{SO}_{0}(p, p-1) .
$$

One consequence is a dichotomy for polystable $\mathrm{SO}(p, q)$-Higgs bundles which translated across the Non-abelian Hodge correspondence leads to the following dichotomy for surface group representations into $\mathrm{SO}(p, q)$.

Theorem 6.9. Let $S$ be a closed surface of genus $g \geq 2$. For $2<p<q-1$, the moduli space $\mathcal{R}(\mathrm{SO}(p, q))$ is a disjoint union of two sets,

$$
\mathcal{R}(\mathrm{SO}(p, q))=\mathcal{R}^{c p t}(\mathrm{SO}(p, q)) \sqcup \mathcal{R}^{e x}(\mathrm{SO}(p, q)),
$$

where 
- $[\rho] \in \mathcal{R}^{\text {cpt }}(\mathrm{SO}(p, q))$ if and only if $\rho$ can be continuously deformed to a compact representation,

- $[\rho] \in \mathcal{R}^{e x}(\mathrm{SO}(p, q))$ if and only if $\rho$ can be continuously deformed to a representation

$$
\rho^{\prime}=\alpha \oplus\left(\iota \circ \rho_{\text {Fuch }}\right) \otimes \operatorname{det}(\alpha),
$$

where $\alpha$ is a representation of $\pi_{1}(S)$ into the compact group $\mathrm{O}(q-p+1)$, $\rho_{\text {Fuch }}$ is a Fuchsian representation of $\pi_{1}(S)$ into $\mathrm{SO}_{0}(2,1)$, and $\iota$ is the principal embedding from (6.17).

Remark 6.10. For $2<p=q-1$, the above theorem does not hold. Namely, there are exactly $2 p(g-1)$ exotic components of $\mathcal{R}(\mathrm{SO}(p, p+1))$ for which the result fails. In [Col17] Collier conjectures that, with the exception of the Hitchin component, all representations in these components are Zariski dense.

\section{Positivity and Cayley correspondence}

7.1. Anosov and positive representations. Anosov representations were introduced by Labourie [Lab06] and have many interesting geometric and dynamic properties. Important examples of Anosov representations include Fuchsian representations, quasiFuchsian representations, Hitchin representations into split real groups and maximal representations into Lie groups of Hermitian type. We briefly describe the main properties of Anosov representations and refer the reader to [Lab06, GW12].

Let $G$ be a semisimple Lie group and $P \subset G$ be a parabolic subgroup. Let $L \subset P$ be the Levi factor of $P$, it is given by $L=P \cap P^{\mathrm{opp}}$, where $P^{\text {opp }}$ is the opposite parabolic of $P$. The homogeneous space $G / L$ is the unique open $G$ orbit in $G / P \times G / P$, and points $(x, y) \in G / P \times G / P$ in this open orbit are called transverse.

Definition 7.1. Let $S$ be closed surface of genus $g \geq 2$. Let $\partial_{\infty} \pi_{1}(S)$ be the Gromov boundary of $\pi_{1}(S)$. Topologically $\partial_{\infty} \pi_{1}(S) \cong \mathbb{R P}^{1}$. A representation $\rho: \pi_{1}(S) \rightarrow G$ is $P$-Anosov if there exists a unique continuous boundary map $\xi_{\rho}: \partial_{\infty} \pi_{1}(S) \rightarrow G / P$ satisfying

- Equivariance: $\xi(\gamma \cdot x)=\rho(\gamma) \cdot \xi(x)$ for all $\gamma \in \pi_{1}(S)$ and all $x \in \partial_{\infty} \pi_{1}(S)$.

- Transversality: for all distinct $x, y \in \partial_{\infty} \pi_{1}(S)$ the generalized flags $\xi(x)$ and $\xi(y)$ are transverse.

- Dynamics preserving: see [Lab06, GW12] for the precise notion.

The map $\xi_{\rho}$ will be called the P-Anosov boundary curve.

One important property of Anosov representations is that they define an open subset of the moduli space of representations $\mathcal{R}(G)$. The set of Anosov representations is however not closed. For example, for the group $\operatorname{PSL}(2, \mathbb{C})$ the set of Anosov representations corresponds to the non-closed set of quasi-Fuchsian representations of $\mathcal{R}(\operatorname{PSL}(2, \mathbb{C}))$. The special cases of Hitchin representations and maximal representations define connected components of Anosov representations. Both Hitchin representations and maximal representations satisfy an additional "positivity" property which is a closed condition. For Hitchin representations this was proved by Labourie [Lab06] and Fock-Goncharov [FG06], and for maximal representations by Burger-Iozzi-Wienhard 
[BIW10]. These notions of positivity have recently been unified and generalized by Guichard and Wienhard [GW16].

For a parabolic subgroup $P \subset G$, denote the Levi factor of $P$ by $L$ and the unipotent subgroup by $U \subset P$. The Lie algebra $\mathfrak{p}$ of $P$ admits an $A d_{L}$-invariant decomposition $\mathfrak{p}=\mathfrak{l} \oplus \mathfrak{u}$ where $\mathfrak{l}$ and $\mathfrak{u}$ are the Lie algebras of $L$ and $U$ respectively. Moreover, the unipotent Lie algebra $\mathfrak{u}$ decomposes into irreducible $L$-representation:

$$
\mathfrak{u}=\bigoplus \mathfrak{u}_{\beta}
$$

Recall that a parabolic subgroup $P$ is determined by fixing a simple restricted root system $\Delta$ of a maximal $\mathbb{R}$-split torus of $G$, and choosing a subset $\Theta \subset \Delta$ of simple roots. To each simple root $\beta_{j} \in \Theta$ there is a corresponding irreducible $L$-representation space $\mathfrak{u}_{\beta_{j}}$.

In [GW16] Guichard and Wienhard introduce the following notions. A pair $\left(G, P^{\Theta}\right)$ admits a positive structure if for all $\beta_{j} \in \Theta$, the $L^{\Theta}$-representation space $\mathfrak{u}_{\beta_{j}}$ has an $L_{0}^{\Theta}$-invariant acute convex cone $c_{\beta_{j}}^{\Theta}$, where $L_{0}^{\Theta}$ denotes the identity component of $L^{\Theta}$.

If $\left(G, P^{\Theta}\right)$ admits a positive structure, then exponentiating certain combinations of elements in the $L_{0}^{\Theta}$-invariant acute convex cones give rise to a semigroup $U_{>0}^{\Theta} \subset U^{\Theta}$. The existence of the semigroup $U_{>0}^{\Theta}$ gives a well defined notion of positively oriented triples of pairwise transverse points in $G / P^{\Theta}$. This notion allows one to define a positive Anosov representation.

If the pair $\left(G, P^{\Theta}\right)$ admits a positive structure, then a $P^{\Theta}$-Anosov representation $\rho: \pi_{1}(S) \rightarrow \mathrm{G}$ is called positive if the Anosov boundary curve $\xi: \partial_{\infty} \pi_{1}(S) \rightarrow G / P^{\Theta}$ sends positively ordered triples in $\partial_{\infty} \pi_{1}(S)$ to positive triples in $G / P^{\Theta}$.

Guichard and Wienhard [GW16] conjecture that if $\left(G, P^{\Theta}\right)$ admits a notion of positivity, then the set $P^{\Theta}$-positive Anosov representations is an open and closed subset of $R(G)$. In particular, the aim of this conjecture is to characterize the connected components of $\mathcal{R}(G)$ which are not labeled by primary topological invariants as being connected components of positive Anosov representations. This is what defines the higher Teichmüller components.

When $G$ is a split real form and $\Theta=\Delta$, the corresponding parabolic is a Borel subgroup of $G$. In this case, the connected component of the identity of the Levi factor is $L_{0}^{\Delta} \cong\left(\mathbb{R}^{+}\right)^{\mathrm{rk}(\mathrm{G})}$ and each simple root space $\mathfrak{u}_{\beta_{i}}$ is one dimensional. The $L_{0}^{\Delta}$-invariant acute convex cone in each simple root space $u_{\beta_{i}}$ is isomorphic to $\mathbb{R}^{+}$. The set of $P^{\Delta}$-positive Anosov representations into a split group are exactly Hitchin representations. When $G$ is a Hermitian Lie group of tube type and $P$ is the maximal parabolic associated to the Shilov boundary of the Riemannian symmetric space of $G$, the pair $(G, P)$ also admits a notion of positivity [BIW10]. In this case, the space of maximal representations into $G$ are exactly the $P$-positive Anosov representations. In particular, the above conjecture holds in these two cases.

In general, the group $\mathrm{SO}(p, q)$ is not a split group and not a group of Hermitian type. Nevertheless, if $p \neq q$, then $\mathrm{SO}(p, q)$ has a parabolic subgroup $P^{\Theta}$ which admits a positive structure. Here $P^{\Theta}$ is the stabilizer of the partial flag $V_{1} \subset V_{2} \subset \cdots \subset V_{p-1}$, where $V_{j} \subset \mathbb{R}^{p+q}$ is a $j$-plane which is isotropic with respect to a signature $(p, q)$ inner product with $p<q$. Here the subgroup $L_{\text {pos }}^{\Theta} \subset L^{\Theta} \subset \mathrm{SO}(p, q)$ which preserves the cones 
$c_{\beta_{j}}^{\Theta}$ is isomorphic to $L_{\text {pos }}^{\Theta} \cong\left(\mathbb{R}^{+}\right)^{l} \times \mathrm{SO}(1, q-p+1)$ with $l=p-1$, We refer the reader to [GW16] and [Col17] for more details.

Using work by Collier [Col17], the following is shown in $[\mathrm{ABC}+18 \mathrm{~b}]$.

Proposition 7.2. Let $P^{\Theta} \subset \mathrm{SO}(p, q)$ be the stabilizer of the partial flag $V_{1} \subset V_{2} \subset \cdots \subset$ $V_{p-1}$, where $V_{j} \subset \mathbb{R}^{p+q}$ is a $j$-plane which is isotropic with respect to a signature $(p, q)$ inner product with $p<q$. If $q>p+1$, then each connected component of $\mathcal{R}^{\text {ex }}(\operatorname{SO}(p, q))$ from (6.18) contains $P^{\Theta}$-positive Anosov representations.

When $q=p+1$, this was shown in [Col17] for the Collier components mentioned in Section 6.3.

Proposition 7.2 gives further evidence for Guichard and Wienhard conjecture, and it is thus natural to expect that all representations in the connected components from Theorem 6.8 are positive Anosov representations. Indeed, this would follow from the conjecture and Proposition 7.2. Moreover, if the conjecture is true, then the connected components of Theorem 6.8 correspond exactly to those connected components of $\mathcal{R}(\mathrm{SO}(p, q))$ which contain positive Anosov representations.

7.2. General Cayley correspondence. As we have seen in Section 5.4, if $G$ is a Hermitian group of tube type, subject to a certain topological constraint (always satisfied if $G$ is of adjoint type, for example), there is a bijective correspondence between maximal $G$-Higgs bundles and $K^{2}$-twisted $H^{*}$-Higgs bundles, where $H^{*}$ is a non-compact group determined by the Shilov boundary. In fact $H^{*}$, is the Levi factor of the parabolic subgroup $P$ which defines positivity and determines the Shilov boundary as $G / P$. In particular, Theorem 5.18 states that the moduli space of maximal $G$-Higgs bundles is isomorphic to the moduli space of $K^{2}$-twisted $H^{*}$-Higgs bundles.

We have also seen in Theorem 6.8 that for the components of the moduli space of $\mathrm{SO}(p, q)$-Higgs bundles containing positive representations, for the positive structure defined above, there is also a generalized Cayley correspondence. Although this seems a bit more involved that the Hermitian case, as explained in $[\mathrm{ABC}+18 \mathrm{~b}]$, the structure of the Cayley partner is entirely determined by the parabolic subgroup of $\mathrm{SO}(p, q)$ defining positivity.

When $G$ is a split real form the Hitchin components of $\mathcal{M}(G)$ admit a similar interpretation. The positivity condition in this case is defined by a minimal parabolic subgroup $P$. The Levi factor is $L=\left(\mathbb{R}^{*}\right)^{\mathrm{rk}(G)}$ and the identity component is $L_{0}=\left(\mathbb{R}^{+}\right)^{\mathrm{rk}(G)}$. Recall from Section 4.3 that the Hitchin base is given $B(G)=\oplus_{i=1}^{r} H^{0}\left(X, K^{d_{i}}\right)$, where $r=\operatorname{rk}(G)$. Now, the summand $H^{0}\left(X, K^{d_{i}}\right)$ can be interpreted as the moduli space of $K^{d_{i}}$-twisted $\mathbb{R}^{+}$-Higgs bundles and the Hitchin components are given by

$$
\mathcal{M}_{K^{d_{1}}}\left(\mathbb{R}^{+}\right) \times \cdots \times \mathcal{M}_{K^{d_{r}}}\left(\mathbb{R}^{+}\right) .
$$

From these three situations it seems natural to conjecture that, in general, higher Teichmüller components, that is, those consisting of positive representations for a certain pair $(G, P)$, are in correspondence with what one can call Cayley components, i.e., components of $\mathcal{M}(G)$ for which there is a Cayley correspondence. Moreover, the structure of the Cayley partner is closely related to the parabolic subgroup defining positivity. Work in this direction is being pursued in [BCGGO], building upon results 
in $[\mathrm{BCGT}]$. This includes the exceptional groups for which there is a notion of positivity. As shown in [GW16] these are real forms of $F_{4}, E_{6}, E_{7}$ and $E_{8}$ whose restricted root system is of type $F_{4}$.

\section{ApPendix A. TABLES}

We use the following notation for Table 2:

- $\Delta_{10}^{ \pm}$are the half-spinor representations of the group $\operatorname{Spin}(10, \mathbb{C})$. They are 16 dimensional.

- $M$ and $M^{*}$ are the irreducible 27-dimensional representations of $\mathrm{E}_{6}$, which are dual to each other.

- $\eta^{r}$ is the representation $\eta^{r}: \mathbb{C}^{*} \rightarrow \mathbb{C}^{*}$ given by $z \mapsto z^{r}$.

\begin{tabular}{|l|l|l|}
\hline Type & $\mathfrak{g}$ & $\widehat{\mathfrak{g}}$ \\
\hline AI & $\mathfrak{s l}(n, \mathbb{R})$ & $\mathfrak{s l}(n, \mathbb{R})$ \\
\hline AII & $\mathfrak{s u}(2 n)$ & $\mathfrak{s l}(n, \mathbb{R})$ \\
\hline \multirow{2}{*}{ AIII } & $\mathfrak{s u}(p, q), p<q$ & $\mathfrak{s o}(p, p+1)$ \\
\cline { 2 - 3 } & $\mathfrak{s u}(p, p)$ & $\mathfrak{s p}(2 p, \mathbb{R})$ \\
\hline \multirow{2}{*}{ BI } & $\mathfrak{s o}(2 p, 2 q+1), p \leq q$ & $\mathfrak{s o}(2 p, 2 p+1)$ \\
\hline CI & $\mathfrak{s p}(2 n, \mathbb{R})$ & $\mathfrak{s p}(2 n, \mathbb{R})$ \\
\hline \multirow{2}{*}{ CII } & $\mathfrak{s p}(p, q) p<q$ & $\mathfrak{s o}(p, p+1)$ \\
\cline { 2 - 3 } & $\mathfrak{s p}(p, p)$ & $\mathfrak{s p}(2 p, \mathbb{R})$ \\
\hline \multirow{2}{*}{ BDI } & $\mathfrak{s o}(p, q) p+q=2 n, p<q$ & $\mathfrak{s o}(p, p+1)$ \\
\hline \multirow{2}{*}{ DI } & $\mathfrak{s o}(p, p)$ & $\mathfrak{s o}(p, p)$ \\
\hline \multirow{2}{*}{ DII } & $\mathfrak{s o *}(4 p+2) p<q$ & $\mathfrak{s o}(p, p+1)$ \\
\cline { 2 - 3 } & $\mathfrak{s o}(4 p)$ & $\mathfrak{s p}(2 p, \mathbb{R})$ \\
\hline
\end{tabular}

\begin{tabular}{|l|l|l|}
\hline Type & $\mathfrak{g}$ & $\widehat{\mathfrak{g}}$ \\
\hline EI & $\mathfrak{e}_{6(6)}$ & $\mathfrak{e}_{6(6)}$ \\
\hline EII & $\mathfrak{e}_{6(2)}$ & $\mathfrak{f}_{4(4)}$ \\
\hline EIII & $\mathfrak{e}_{6(-14)}$ & $\mathfrak{s o}(3,2)$ \\
\hline EIV & $\mathfrak{e}_{6(-26)}$ & $\mathfrak{s l}(3, \mathbb{R})$ \\
\hline EV & $\mathfrak{e}_{7(7)}$ & $\mathfrak{e}_{7(7)}$ \\
\hline EVI & $\mathfrak{e}_{7(-5)}$ & $\mathfrak{f}_{4(4)}$ \\
\hline EVII & $\mathfrak{e}_{7(-25)}$ & $\mathfrak{s p}_{(6, \mathbb{R})}$ \\
\hline EVIII & $\mathfrak{e}_{8(8)}$ & $\mathfrak{e}_{8(8)}$ \\
\hline EIX & $\mathfrak{e}_{8(-24)}$ & $\mathfrak{f}_{4(4)}$ \\
\hline FI & $\mathfrak{f}_{4(4)}$ & $\mathfrak{f}_{4(4)}$ \\
\hline FII & $\mathfrak{f}_{4(-20)}$ & $\mathfrak{s l}_{(2, \mathbb{R})}$ \\
\hline G & $\mathfrak{g}_{2(2)}$ & $\mathfrak{g}_{2(2)}$ \\
\hline
\end{tabular}

TABLE 1. Maximal split subalgebras 


\begin{tabular}{|c|c|c|c|}
\hline$G$ & $H$ & $H^{\mathbb{C}}$ & $\mathfrak{m}^{\mathbb{C}}=\mathfrak{m}^{+}+\mathfrak{m}^{-}$ \\
\hline \hline $\mathrm{SU}(p, q)$ & $\mathrm{S}(\mathrm{U}(p) \times \mathrm{U}(q))$ & $\mathrm{S}(\mathrm{GL}(p, \mathbb{C}) \times \mathrm{GL}(q, \mathbb{C}))$ & $\operatorname{Hom}\left(\mathbb{C}^{q}, \mathbb{C}^{p}\right)+\operatorname{Hom}\left(\mathbb{C}^{p}, \mathbb{C}^{q}\right)$ \\
\hline $\mathrm{Sp}(2 n, \mathbb{R})$ & $\mathrm{U}(n)$ & $\mathrm{GL}(n, \mathbb{C})$ & $S^{2}\left(\mathbb{C}^{n}\right)+S^{2}\left(\mathbb{C}^{n *}\right)$ \\
\hline $\mathrm{SO}^{*}(2 n)$ & $\mathrm{U}(n)$ & $\mathrm{GL}(n, \mathbb{C})$ & $\Lambda^{2}\left(\mathbb{C}^{n}\right)+\Lambda^{2}\left(\mathbb{C}^{n *}\right)$ \\
\hline $\mathrm{SO}_{0}(2, n)$ & $\mathrm{SO}(2) \times \mathrm{SO}(n)$ & $\mathrm{SO}(2, \mathbb{C}) \times \mathrm{SO}(n, \mathbb{C})$ & $\operatorname{Hom}\left(\mathbb{C}^{n}, \mathbb{C}\right)+\operatorname{Hom}\left(\mathbb{C}, \mathbb{C}^{n}\right)$ \\
\hline $\mathrm{E}_{6}^{-14}$ & $\mathrm{Spin}(10) \times_{\mathbb{Z}_{4}} \mathrm{U}(1)$ & $\mathrm{Spin}(10, \mathbb{C}) \times_{\mathbb{Z}_{4}} \mathbb{C}^{*}$ & $\Delta_{10}^{+} \otimes \eta^{3}+\Delta_{10}^{-} \otimes \eta^{-3}$ \\
\hline $\mathrm{E}_{7}^{-25}$ & $\mathrm{E}_{6}^{-78} \times_{\mathbb{Z}_{3}} \mathrm{U}(1)$ & $\mathrm{E}_{6} \times_{\mathbb{Z}_{3}} \mathbb{C}^{*}$ & $M \otimes \eta^{2}+M^{*} \otimes \eta^{-2}$ \\
\hline
\end{tabular}

TABLE 2. Irreducible Hermitian symmetric spaces $G / H$

\begin{tabular}{|c|c|c|c|c|c|c|}
\hline$G$ & $H$ & $N$ & $\operatorname{dim} \mathfrak{m}$ & $\ell$ & $o\left(e^{2 \pi J}\right)$ & $q_{T}$ \\
\hline \hline $\mathrm{SU}(p, q)$ & $\mathrm{S}(\mathrm{U}(p) \times \mathrm{U}(q))$ & $p+q$ & $2 p q$ & $l c m(p, q)$ & $\frac{p+q}{g c d(p, q)}$ & $1 / 2$ \\
\hline $\mathrm{Sp}(2 n, \mathbb{R})$ & $\mathrm{U}(n)$ & $n+1$ & $n(n+1)$ & $n$ & 2 & $1 / 2$ \\
\hline $\mathrm{SO}^{*}(2 n)$ & $\mathrm{U}(n)$ & $2(n-1)$ & $n(n-1)$ & $n$ & 2 & 1 \\
\hline $\mathrm{SO}_{0}(2, n)$ & $\mathrm{SO}(2) \times \mathrm{SO}(n)$ & $n$ & $2 n$ & 1 & 1 & $1 / 2$ \\
\hline $\mathrm{E}_{6}^{-14}$ & $\mathrm{Spin}(10) \times_{\mathbb{Z}_{4}} \mathrm{U}(1)$ & 12 & 32 & 4 & 3 & $1 / 2$ \\
\hline $\mathrm{E}_{7}^{-25}$ & $\mathrm{E}_{6}^{-78} \times_{\mathbb{Z}_{3}} \mathrm{U}(1)$ & 18 & 54 & 3 & 2 & $1 / 2$ \\
\hline
\end{tabular}

TABLE 3. Toledo character data for the classical and exceptional groups

\begin{tabular}{|c|c|c|c|c|c|c|}
\hline$G$ & $H$ & $N$ & $\operatorname{dim} \mathfrak{m}$ & $\ell$ & $o\left(e^{2 \pi J}\right)$ & $q_{T}$ \\
\hline $\mathrm{PSU}(p, q)$ & $\mathrm{PS}(\mathrm{U}(p) \times \mathrm{U}(q))$ & $p+q$ & $2 p q$ & $g c d(p, q)$ & 1 & $\frac{p+q}{2 l c m(p, q)}$ \\
\hline$P \mathrm{SO}^{*}(2 n=4 m+2)$ & $\mathrm{U}(n)$ & $2(n-1)$ & $n(n-1)$ & $n$ & 1 & 2 \\
\hline $\mathrm{E}_{6}^{-14} / \mathbb{Z}_{3}$ & $\operatorname{Spin}(10) \times_{\mathbb{Z}_{4}} \mathrm{U}(1)$ & 12 & 32 & 4 & 1 & $3 / 2$ \\
\hline
\end{tabular}

TABLE 4. Toledo character data for adjoint groups of non-tube type. 


\begin{tabular}{|c|c|c|c|c|c|c|}
\hline$G$ & $H$ & $H^{*}$ & $H^{\prime}$ & $\check{S}=H / H^{\prime}$ & $\mathfrak{m}^{\prime}$ & $\mathfrak{m}^{\prime \mathbb{C}}$ \\
\hline $\mathrm{SU}(n, n)$ & $\mathrm{S}(\mathrm{U}(n) \times \mathrm{U}(n))$ & $\begin{array}{l}\{A \in \operatorname{GL}(n, \mathbb{C}) \mid \\
\left.\quad \operatorname{det}(A)^{2} \in \mathbb{R}^{+}\right\}\end{array}$ & $\begin{array}{l}\{A \in \mathrm{U}(n) \mid \\
\left.\operatorname{det}(A)^{2}=1\right\}\end{array}$ & $\mathrm{U}(n)$ & $\operatorname{Herm}(n, \mathbb{C})$ & $\operatorname{Mat}(n, \mathbb{C})$ \\
\hline $\operatorname{Sp}(2 n, \mathbb{R})$ & $\mathrm{U}(n)$ & $\operatorname{GL}(n, \mathbb{R})$ & $\mathrm{O}(n)$ & $\mathrm{U}(n) / \mathrm{O}(n)$ & $\operatorname{Sym}(n, \mathbb{R})$ & $\operatorname{Sym}(n, \mathbb{C})$ \\
\hline $\begin{array}{l}\mathrm{SO}^{*}(2 n) \\
n=2 m\end{array}$ & $\mathrm{U}(n)$ & $\mathrm{U}^{*}(n)$ & $\operatorname{Sp}(n)$ & $\mathrm{U}(n) / \mathrm{Sp}(n)$ & $\operatorname{Herm}(m, \mathbb{H})$ & $\operatorname{Skew}(n, \mathbb{C})$ \\
\hline $\mathrm{SO}_{0}(2, n)$ & $\mathrm{SO}(2) \times \mathrm{SO}(n)$ & $\mathrm{SO}_{0}(1,1) \times \mathrm{SO}(1, n-1)$ & $\mathrm{O}(n-1)$ & $\frac{\mathrm{U}(1) \times S^{n-1}}{\mathbb{Z}_{2}}$ & $\mathbb{R} \times \mathbb{R}^{n-1}$ & $\mathbb{C} \times \mathbb{C}^{n-1}$ \\
\hline $\mathrm{E}_{7}^{-25}$ & $\mathrm{E}_{6}^{-78} \times_{\mathbb{Z}_{3}} \mathrm{U}(1)$ & $\mathrm{E}_{6}^{-26} \ltimes \mathbb{R}^{*}$ & $\mathrm{~F}_{4} \times \mathbb{Z}_{2}$ & $\frac{\mathrm{E}_{6}^{-78} \cdot \mathrm{U}(1)}{\mathrm{F}_{4}}$ & $\operatorname{Herm}(3, \mathbb{O})$ & $\operatorname{Herm}(3, \mathbb{O}) \otimes \mathbb{C}$ \\
\hline
\end{tabular}

TABLE 5. Irreducible Hermitian symmetric spaces $G / H$ of tube type 


\section{REFERENCES}

[ABC+18] M. Aparicio-Arroyo, S.B. Bradlow, B. Collier, O. García-Prada, P.B. Gothen and A. Oliveira, Exotic components of $\mathrm{SO}(p, q)$ surface group representations and their Higgs bundle avatars, C.R. Math. Acad. Sci. Paris 356 (2018) 666-673.

$[\mathrm{ABC}+18 \mathrm{~b}] \_, \mathrm{SO}(p, q)$-Higgs bundles and higher Teichmüller components, arXiv:1802.08093, 2018.

[AB83] M. F. Atiyah and R. Bott The Yang-Mills equations over Riemann surfaces. Philos. Trans. Roy. Soc. London Ser. A, 308 (1983) 523-615.

[BCGT] O. Biquard, B. Collier, O. García-Prada and D. Toledo, Arakelov-Milnor inequalities and maximal variations of Hodge structure. In preparation.

[BGM15] O. Biquard, O. García-Prada and I. Mundet i Riera, Parabolic Higgs bundles and representations of the fundamental group of a puntured surface into a real group, arXiv: 1510.042207.

[BGR17] O. Biquard, O. García-Prada and R. Rubio, Higgs bundles, the Toledo invariant and the Cayley correspondence, J. of Topology 10 (2017) 795-826.

[BCGGO] S.B. Bradlow, B. Collier, O. García-Prada, P.B. Gothen and A. Oliveira, Higher Teichmüller spaces, positivity and Cayley correspondence. In preparation.

[BGG03] S.B. Bradlow, O. García-Prada, and P.B. Gothen. Surface group representations and U(p,q)-Higgs bundles. J. Differential Geom. 64 (2003) 111-170.

[BGG06] Maximal surface group representations in isometry groups of classical Hermitian symmetric spaces. Geom. Dedicata, 122 (2006) 185-213.

[BGG13] Deformations of maximal representations in $\operatorname{Sp}(4, \mathbb{R})$, Q. J. Math. 63 (2012) 795-843.

[BGG15] - Higgs bundles for the non-compact dual of the special orthogonal group Geom. Dedicata 175 (2015) 1-48.

[BGM03] S. B. Bradlow, O. García-Prada, and I. Mundet i Riera, Relative Hitchin-Kobayashi correspondences for principal pairs, Quarterly J. Math., 54 (2003) 171-208.

[BILW05] M. Burger, A. Iozzi, F. Labourie, and A. Wienhard, Maximal representations of surface groups: symplectic Anosov structures, Pure Appl. Math. Q. 1 (2005) 543-590.

[BIW10] M. Burger, A. Iozzi, and A. Wienhard. Surface group representations with maximal Toledo invariant. Ann. of Math. (2) 172 (2010) 517-566.

[BIW14] M. Burger, A. Iozzi, and A. Wienhard, Higher Teichmüller Spaces: from SL $(2, \mathbb{R})$ to other Lie groups. In Handbook of Teichmüller theory, Vol. IV, volume 19 of IRMA Lect. Math. Theor. Phys., pages 539-618. EMS, Zürich 2014

[ChG93] S. Choi and W.M. Goldman, Convex real projective structures on closed surfaces are closed Proc. Amer. Math. Soc.118 (1993) 657-661.

[CO03] J.-L. Clerc and B. Orsted, The Gromov norm of the Kaehler class and the Maslov index Asian J. Math., 7 (2003) 269-295.

[Col17] B. Collier, $\mathrm{SO}(n, n+1)$-surface group representations and their Higgs bundles, arXiv:1710.01287, 2017.

[CTT17] B. Collier, N. Tholozan, J. Toulisse, The geometry of maximal representations of surface groups into $\mathrm{SO}(2, n)$, arXiv: 1702.08799, 2017.

[Cor88] K. Corlette, Flat G-bundles with canonical metrics, J. Differential Geom., 28 (1988) 361-382.

[DG01] R.Y. Donagi and D. Gaitsgory, The gerbe of Higgs bundles, Transform. Groups, 7 (2001) 109-153.

[Don87] S.K. Donaldson, Twisted harmonic maps and the self-duality equations. Proc. London Math. Soc. (3) 55 (1987) 127-131.

[DT87] A. Domic and D. Toledo, The Gromov norm of the Kaehler class of symmetric domains. Math. Ann. 276 (1987) 425-432.

[Dup78] J. L. Dupont, Bounds for characteristic numbers of flat bundles, Springer LNM, 763 (1978). 
[FK94] J. Faraut and A. Korányi. Analysis on symmetric cones. Oxford Mathematical Monographs. The Clarendon Press Oxford University Press, New York, 1994. Oxford Science Publications.

[FG06] V.V. Fock and A.B. Goncharov, Moduli spaces of local systems and higher Teichmüller theory, Inst. Hautes Études Sci. Publ. Math. 103 (2006) 1-211.

[GGM09] O. García-Prada, P. B. Gothen, and I. Mundet i Riera. The Hitchin-Kobayashi correspondence, Higgs pairs and surface group representations, arXiv:0909.4487.

[GGM13] Higgs bundles and surface group representations in the real symplectic group, Journal of Topology, 6 (2013) 64-118.

[GM04] O. García-Prada and I. Mundet i Riera, Representations of the fundamental group of a closed oriented surface in $\mathrm{Sp}(4, \mathbb{R})$, Topology, 43 (2004) 831-855.

[GO14] O. García-Prada and A. Oliveira, Connectedness of Higgs bundle moduli for complex reductive Lie groups, Asian Journal of Mathematics, 21 (2017) 791-810.

[GP19] O. García-Prada and A. Peón-Nieto, Higgs pairs, abelian gerbes and cameral data, preprint 2019.

[GPR18] O. García-Prada, A. Peón-Nieto and S. Ramanan, Higgs bundles for real groups and the Hitchin-Kostant-Rallis section Transactions of the AMS 370 (2018) 2907-2953.

[GR18] O. García-Prada and S. Ramanan, Involutions of rank 2 Higgs bundle moduli spaces. In Geometry and Physics: A Festschrift in honour of Nigel Hitchin, Oxford University Press, 2018.

[GW08] O. Guichard and A. Wienhard, Convex foliated projective structures and the Hitchin component for PSL $(4, \mathbb{R})$, Duke Math. J. 144 (2008) 381-445.

[GW12] O. Guichard and A. Wienhard, Anosov representations: domains of discontinuity and applications Invent. Math. 190 (2012) 357-438.

[GW16] O. Guichard and A. Wienhard, Positivity and higher Teichmüller theory, Proceedings of the 7th European Congress of Mathematics, 2016.

[Gol88] W.M. Goldman, Topological components of spaces of representations. Invent. Math. 93 (1988) 557-607.

[Got01] P. B. Gothen, Components of spaces of representations and stable triples, Topology 40 (2001) 823-850.

[Hel01] S. Helgason, Differential geometry, Lie groups, and symmetric spaces, volume 34 of Graduate Studies in Mathematics. American Mathematical Society, Providence, RI, 2001. Corrected reprint of the 1978 original.

[Her91] L. Hernández, Maximal representations of surface groups in bounded symmetric domains Trans. Amer. Math. Soc., 324 (1991) 405-420.

[Hit87] N. J. Hitchin, The self-duality equations on a Riemann surface, Proc. London Math. Soc., 55 (1987) 59-126.

[Hit87b] _ Stable bundles and integrable systems, Duke Math. J. 54 (1987) 91-114.

[Hit92] L Lie groups and Teichmüller space. Topology, 31 (1992) 449-473.

[HS14] N. J. Hitchin and L. Schaposnik, Nonabelianization of Higgs bundles, J. Differential Geom. 97, (2014) 79-89.

[Kna96] A. W. Knapp, Lie Groups beyond an Introduction, first ed., Progress in Mathematics, vol 140, Birkhäuser Boston Inc., Boston, MA, 1996.

[KP14] I. Kim and P. Pansu, Density of Zariski density for surface groups, Duke Math. J. 163 (2014) 1737-1794.

[Kob87] S. Kobayashi. Differential geometry of complex vector bundles, volume 15 of Publications of the Mathematical Society of Japan. Princeton University Press, Princeton, NJ, 1987. Kanô Memorial Lectures, 5 .

[KV79] A. Korányi and S. Vági. Rational inner functions on bounded symmetric domains. Trans. Amer. Math. Soc., 254 (1979) 179-193.

[KR71] B. Kostant and S. Rallis, Orbits and representations associated with symmetric spaces, Amer. J. Math. 93 (1971) 753-809. 
[Lab06] F. Labourie, Anosov flows, surface groups and curves in projective space, Invent. Math. 165 (2006) 51-114.

[Li93] J. Li, The Space of Surface Group Representations, Manuscripta Math. 78 (1993), 223243.

[Mil58] J. W. Milnor, On the existence of a connection with curvature zero, Commm. Math. Helv. 32 (1958) 215-223.

[Nit91] N. Nitsure, Moduli space of semistable pairs on a curve, Proc. London Math. Soc. 62 (1991) 275-300.

[Peo13] A. Peón-Nieto, Higgs bundles, real forms and the Hitchin fibration, Ph.D. Thesis, UAMICMAT, Madrid 2013.

[Ram96] A. Ramanathan. Moduli for principal bundles over algebraic curves: I and II, Proc. Indian Acad. Sci. Math. Sci. 106 (1996) 301-328 and 421-449.

[Sch08] A. H. W. Schmitt. Geometric invariant theory and decorated principal bundles. Zurich Lectures in Advanced Mathematics. European Mathematical Society (EMS), Zürich, 2008.

[Sim88] C. T. Simpson, Constructing variations of Hodge structure using Yang-Mills theory and applications to uniformization, J. Amer. Math. Soc. 1 (1988) 867-918.

[Sim90] C. T. Simpson, Harmonic bundles of non-compact curves J. Amer. Math. Soc. 3 (1990) 713-770.

[Sim92] _ Higgs bundles and local systems, Inst. Hautes Études Sci. Publ. Math. 75 (1992), $5-95$.

[Sim94] Moduli of representations of the fundamental group of a smooth projective variety I, Publ. Math., Inst. Hautes Étud. Sci. 79 (1994) 47-129.

[Sim95] Moduli of representations of the fundamental group of a smooth projective variety II, Publ. Math., Inst. Hautes Étud. Sci. 80 (1995) 5-79.

[Sch13] L.P. Schaposnik, Spectral data for G-Higgs bundles, DPhil thesis, University of Oxford, 2013.

[Tol89] D. Toledo. Representations of surface groups in complex hyperbolic space. J. Differential Geom., 29 (1989) 125-133.

[Wien18] A. Wienhard, An invitation to higher Teichmüller theory, Proc. Int. Cong. of Math. Rio de Janeiro, Vol. 1 (2018), 1007-1034.

[Woo71] J. W. Wood, Bundles with totally disconnected structure group, Comment. Math. Helv. 46 (1971) 257-273.

Instituto de Ciencias Matemáticas, CSIC-UAM-UC3M-UCM, Nicolás Cabrera, 13-15, 28049 MADRID, SPAIN

E-mail address: oscar.garcia-prada@icmat.es 